\title{
NJL model on the light cone and pion structure function *
}

\author{
W. Bentz, T. Hama, T. Matsuki, K. Yazaki \\ Department of Physics, \\ Faculty of Science \\ University of Tokyo \\ Hongo 7-3-1, Bunkyo-ku, Tokyo 113, Japan
}

\begin{abstract}
The NJL model is formulated on the light cone. Using the $1 / \mathrm{N}$ expansion to solve the fermionic constraint, an effective 4-fermi lagrangian is derived. Pionic properties are investigated using this lagrangian, and the formal equivalence to the equal-time formulation is demonstrated. Two regularization schemes in terms of light-cone variables are discussed: An extension of the 'invariant mass cut-off scheme', and a transverse momentum cut-off scheme. It is shown that the first one is equivalent to the covariant 3-momentum cut-off (dispersion cut-off) scheme in the equal-time formulation. As an application the structure function of the pion is studied in both regularization schemes.
\end{abstract}

PACS numbers:

Keywords: Effective quark theories, light cone theories, structure functions

${ }^{*}$ Correspondence to: W. Bentz, E-mail: bentz@tkynt2.phys.s.u-tokyo. ac.jp 


\section{Introduction}

As an effective theory of QCD in the low energy region, the Nambu - JonaLasinio (NJL) model [1] is a powerful tool to investigate the properties of hadrons [2]. It exhibits the spontaneous breaking of chiral symmetry and the appearance of Goldstone bosons in a simple and clear way. Due to the simplicity of the interaction, the relativistic two- and three-body equations can be solved in the ladder approximation, which allows a consistent unified description of mesons and baryons [3, [1]. Recently, extensions have been made to include also mesonic degrees of freedom into the description of threequark bound states [5], and methods to incorporate the effects of confinement phenomenologically have been proposed [6]. Concerning the ground state properties of baryons, the NJL model has been quite successful [7].

Recently, the model has been used also to describe the structure functions of the pion [8] and the nucleon [9] measured in deep inelastic scattering. It is well known that in the Bjorken limit the structure functions are expressed as Fourier transforms of correlation functions along the light-cone (LC) [10]. Therefore, for the evaluation of the structure functions in the Bjorken limit the use of LC variables is most natural [11]. The interpretation of the structure functions as LC momentum distributions of the constituents (partons) in the ground state of the hadron, however, is strictly speaking possible only in a framework where the LC correlation functions can be considered as equal 'time' correlations. In the LC quantization procedure [11, 12, 13 this is indeed the case, since in this approach the fields evolve in the 'time' variable

$x^{+}=\left(x^{0}+x^{3}\right) / \sqrt{2}$ instead of the usual time variable $x^{0}$. Therefore, from the physical standpoint one expects that the most natural way to describe the 
structure functions in the Bjorken limit consists of not only using LC variables, but also LC quantization. The LC quantization, which was proposed by Dirac |14 a long time ago, has also many other interesting features, like the 'vacuum triviality' [15] and associated with this the fact that Fock space expansions of physical states have an exact meaning, without referring to a particular approximation scheme [16].

In spite of these physically appealing features, LC treatments also have mostly technical - disadvantages. For example, they conceal the underlying rotational invariance and complicate the treatment of angular momentum, and bring in new problems of how to regularize divergent integrals [18]. Moreover, the presence of zero modes destroys the 'vacuum triviality' in the naive sense, and the proper treatment of these zero modes is actually very complicated and technically involved [17]. Therefore, in actual calculations of structure functions in the NJL model, it might eventually be more convenient to use the usual equal time (ET) quantization and merely perform a transformation to LC variables at some convenient stage. Nevertheless, it is an important task to show that such a procedure is indeed equivalent to the LC quantization method with all its physical merits. To investigate this equivalence for the case of the NJL model is the purpose of this work.

The demonstration of the equivalence of ET and LC quantization for $\sigma$-model type theories was the subject of previous investigations [12]. For 4-fermi theories like the NJL model, it is well known that the LC quantization poses additional problems due to the nonlinearity of the fermionic constraint 18, 19. To circumvent this problem, in some previous works 19 this fermionic constraint equation was replaced by the corresponding equation for free fields. In this work we will show a straight forward method 
[20] based on the $1 / N$ expansion up to the next-to-leading order to solve the fermionic constraint. (For methods using boson mapping techniques see ref. [18].) We will show that the resulting effective lagrangian has the same form as obtained by using the free field equations to solve the fermionic constraint, but the 4-fermi coupling constant is replaced by an effective one. For physical quantities, the changes due to this modified coupling constant cancel against those due to modified propagators, such that the theory is formally equivalent to the ET theory. We will show that there exist cut-off schemes where this equivalence holds even for the regularized theories. Namely, a suitably extended version of the "invariant mass cut-off" introduced in ref. [21] will be shown to be equivalent to the ET treatment based on the covariant 3-momentum cut-off (dispersion cut-off) [22]. We will also discuss a recently proposed alternative regularization scheme [23] which has no counterpart in the conventional ET treatment. As an application, we will discuss the pion structure function, and compare our results with the empirically derived valence quark distributions in the pion [24].

The rest of this paper is organized as follows: In sect. 2 we review some well known facts of the NJL model in the ET formulation based on the covariant 3-momentum cut-off scheme, and discuss the pion structure function in the ET formulation. In sect. 3 we derive the effective LC lagrangian in the framework of the 1/N expansion, discuss the new Feynman rules based on the LC quantization, and show the formal equivalence to the ET theory. Two regularization schemes suitable for LC theories will be discussed. In sect. 4 we will show our numerical results for the valence quark LC momentum distribution in the pion, and in sect. 5 we summarize our work. 


\section{NJL model in the ET quantization and the pion structure function}

\subsection{The model}

The lagrangian of the flavor SU(2) NJL model in its original form is given by [1]

$$
\mathcal{L}=\mathcal{L}_{0}+\mathcal{L}_{I}
$$

where $\mathcal{L}_{0}=\bar{\psi}(i \not \partial-m) \psi$ is the free quark lagrangian with $m$ the current $u, d$ quark mass, and $\mathcal{L}_{I}$ is given by

$$
\mathcal{L}_{I}=G\left((\bar{\psi} \psi)^{2}-\left(\bar{\psi}\left(\gamma_{5} \boldsymbol{\tau}\right) \psi\right)^{2}\right)
$$

The gap equation is given as usual by

$$
M=m-2 G\langle\bar{\psi} \psi\rangle=m+12 i G \int \frac{d^{4} q}{(2 \pi)^{4}} \operatorname{tr}_{D}\left(S(q) \gamma^{0}\right) .
$$

$M$ is the constituent quark mass, $S(q)=\frac{1}{\not q-M+i \epsilon} \gamma^{0}$ is the Feynman propagator for the constituent quark 円, and $\langle\bar{\psi} \psi\rangle$ denotes the quark condensate in the leading order $(N)$ of the $1 / N$ expansion. The solution of the BS equation for the $q \bar{q}$ t-matrix in the pionic channel is

$$
t(k)=\left(\gamma^{0} \gamma_{5} \tau_{i}\right) \tau(k)\left(\gamma^{0} \gamma_{5} \tau_{i}\right)
$$

\footnotetext{
${ }^{1}$ To correspond to the conventions used in most papers on LC field theories (see for example ref. [11]), we define the Feynman propagator via the T-product of $\psi$ and $\psi^{+}$, and normalize spinors according to $u^{\dagger}\left(\boldsymbol{p}, s^{\prime}\right) u(\boldsymbol{p}, s)=2 E_{p} \delta_{s^{\prime} s}$. We also restrict ourselves to the lowest order in the $1 / N$ expansion (direct terms only), and therefore the effective coupling constant in the scalar and pseudoscalar channels is given by $G$ (instead of $\frac{13}{12} G$ ). Throughout this paper, $<\bar{\psi} \Gamma \psi>$ denotes the leading order $(N)$ of the vacuum expectation value of $\bar{\psi} \Gamma \psi$.
} 
Here the reduced t-matrix is given by

$$
\tau(k)=\frac{-2 i G}{1+2 G \Pi(k)},
$$

where

$$
\Pi(k)=6 i \int \frac{d^{4} q}{(2 \pi)^{4}} \operatorname{tr}_{D}\left[\gamma^{0} \gamma_{5} S(q) \gamma^{0} \gamma_{5} S(q-k)\right]
$$

is the familiar 'bubble graph'. The pion mass $m_{\pi}$ is obtained as the pole of (2.5).

The Bethe-Salpeter (BS) wave function $\Phi_{k}(q)$ in the covariant normalization is defined via the residue of the connected $q \bar{q}$ Green function at the pion pole $k_{0}=\omega_{k} \equiv \sqrt{\boldsymbol{k}^{2}+m_{\pi}^{2}}$, i.e;

$$
S\left(q^{\prime}\right) S\left(q^{\prime}-k\right) t(k) S(q-k) S(q) \longrightarrow \frac{i}{2 \omega_{k}} \frac{\Phi_{k}^{i}\left(q^{\prime}\right) \Phi_{k}^{i+}(q)}{k^{0}-\omega_{k}+i \epsilon} \quad \text { as } k_{0} \rightarrow \omega_{k},
$$

which leads to

$$
\Phi_{k}^{i}(q)=g\left(S(q) \gamma^{0} \gamma_{5} \tau_{i} S(q-k)\right) ; \quad \frac{1}{g^{2}}=-\left(\frac{\partial \Pi}{\partial k^{2}}\right)_{k^{2}=m_{\pi}^{2}} .
$$

Here $g$ is the pion-quark coupling constant. The assignment of the external momenta in eq. (2.7) is shown in fig. 1.

The pion decay constant is defined as usual via the matrix element of the axial vector current between the pion state $\mid k i>$ and the vacuum:

$$
<0\left|\bar{\psi}(0) \frac{\tau_{j}}{2} \gamma^{\mu} \gamma_{5} \psi(0)\right| k i>=i \delta_{i j} k^{\mu} f_{\pi}
$$

This amounts to calculating the familiar one-quark loop contribution to the pion decay, using the BS wave function eq. (2.8) at the $\bar{q} q \pi$ vertex. The results is

$i k^{\mu} f_{\pi}=3 g \int \frac{d^{4} q}{(2 \pi)^{4}} \operatorname{tr}_{D}\left(\gamma^{0} \gamma^{\mu} \gamma_{5} S(q-k) \gamma^{0} \gamma_{5} S(q)\right)$ 


$$
=12 g M k^{\mu} \int \frac{d^{4} q}{(2 \pi)^{4}} \frac{1}{\left(q^{2}-M^{2}+i \epsilon\right)\left((q-k)^{2}-M^{2}+i \epsilon\right)} \quad\left(k^{2}=m_{\pi}^{2}\right) .
$$

\subsection{Quark LC momentum distribution}

In the ET quantization method, the valence quark LC momentum distribution $q_{v}(x)$ in the pion as a function of the Bjorken variable $x$ [10]

$$
q_{v}(x)=\frac{1}{4 \pi} \int d z^{-} e^{i x k_{-} z^{-}}\left\langle k i\left|\bar{\psi}(0) \gamma^{+} \psi\left(z^{-}\right)\right| k i\right\rangle
$$

can be obtained from the diagram fig. 2), where the external operator is $\gamma^{+}$ and the loop integral is performed with respect to $q_{+}$and $\mathbf{q}_{\perp}$ with $q_{-}=$ $x k_{-}$fixed \&. Using $S(q) \gamma^{0} \gamma^{+} S(q)=-\partial S(q) / \partial q_{+}$and performing a partial integration in $q_{+}$, we get

$$
\begin{aligned}
q_{v}(x) & =3 i g^{2} \int \frac{d q_{+} d^{2} q_{\perp}}{(2 \pi)^{4}} \operatorname{tr}_{D}\left(S(q) \gamma^{0} \gamma^{+} S(q) \gamma^{0} \gamma_{5} S(q-k) \gamma^{0} \gamma_{5}\right) \\
& =-g^{2} \frac{\partial \Pi(k, x)}{\partial k^{2}} \quad\left(k^{2}=m_{\pi}^{2}\right)
\end{aligned}
$$

where

$$
\Pi(k, x)=6 i k_{-} \int \frac{d q_{+} d^{2} q_{\perp}}{(2 \pi)^{4}} \operatorname{tr}_{D}\left(\gamma^{0} \gamma_{5} S(q) \gamma^{0} \gamma_{5} S(q-k)\right)
$$

with $x=\frac{q_{-}}{k_{-}}$. The quantity $(2.15)$ is defined such that

$$
\int_{-\infty}^{\infty} d x \Pi(k, x)=\Pi(k)
$$

To evaluate (2.15), we need the Feynman propagator in terms of LC variables:

$$
S(p)=\frac{\gamma^{+} p_{+}+\gamma^{-} p_{-}-\gamma_{\perp} \cdot \boldsymbol{p}_{\perp}+M}{p^{2}-M^{2}+i \epsilon} \gamma^{0}
$$

\footnotetext{
${ }^{2}$ Our conventions for LC variables are summarized in Appendix A.
} 
where

$$
\frac{1}{p^{2}-M^{2}+i \epsilon}=\frac{1}{2 p_{-}}\left(\frac{\Theta\left(p_{-}\right)}{p_{+}-e_{p}+i \epsilon}+\frac{\Theta\left(-p_{-}\right)}{p_{+}-e_{p}-i \epsilon}\right)
$$

with

$$
e_{p}=\frac{\boldsymbol{p}_{\perp}^{2}+M^{2}}{2 p_{-}} .
$$

The Feynman propagator (2.17) contains a contact term in the LC 'time' variable $x^{+}$(conjugate momentum $p_{+}$), and it is convenient to separate this term:

$$
S(p)=\frac{\bar{p}+M}{p^{2}-M^{2}+i \epsilon} \gamma^{0}+\frac{\gamma^{+} \gamma^{0}}{2 p_{-}} \equiv \bar{S}(p)+\frac{\gamma^{+} \gamma^{0}}{2 p_{-}},
$$

where $\bar{p}^{\mu}$ is the on-shell four momentum with LC components $\left(p_{+}=e_{p}, p_{-}, \boldsymbol{p}_{\perp}\right)$. Inserting this into eq. (2.15) and (2.16), one obtains

$$
\Pi(k, x)=\bar{\Pi}(k, x)+R(x), \quad \Pi(k)=\bar{\Pi}(k)+R,
$$

where $\bar{\Pi}(k, x) \quad(\bar{\Pi}(k))$ is obtained by replacing $S \rightarrow \bar{S}$ in eq. (2.15) (eq. (2.6)). For $\boldsymbol{k}_{\perp}=0$ we obtain

$$
\begin{aligned}
\bar{\Pi}(k, x) & =-\frac{6 \Theta(x) \Theta(1-x)}{x(1-x)} \int \frac{d^{2} q_{\perp}}{(2 \pi)^{3}} \frac{\boldsymbol{q}_{\perp}^{2}+M^{2}}{\boldsymbol{q}_{\perp}^{2}+M^{2}-k^{2} x(1-x)-i \epsilon} \\
\bar{\Pi}(k) & =\int_{0}^{1} d x \bar{\Pi}(k, x),
\end{aligned}
$$

while $R$ is due to the contact term and given by

$$
R=\int_{-\infty}^{\infty} d x R(x)=-3 i \mathcal{P} \int \frac{d^{4} q}{(2 \pi)^{4}} \operatorname{tr}_{D}\left(\frac{\bar{S}(q) \gamma^{0} \gamma^{+}}{q_{-}-k_{-}}+\frac{\bar{S}(q-k) \gamma^{0} \gamma^{+}}{q_{-}}\right),
$$

where $\mathcal{P}$ denotes the principal value with respect to the $q_{-}$integration. Due to eq. (2.20), $\bar{S}$ can be replaced by $S$ in this expression. Using (2.18) and 
performing the $q_{+}$integral we obtain of

$$
R=-3 \mathcal{P} \int_{-\infty}^{\infty} d x\left(\frac{\Theta(x)}{x-1}-\frac{\Theta(-x)}{x-1}+\frac{\Theta(x+1)}{x}-\frac{\Theta(x-1)}{x}\right) \int \frac{d^{2} q_{\perp}}{(2 \pi)^{3}} .
$$

It should be noted that in obtaining the expressions (2.22) and (2.25), no shifts of integration variables have been performed.

The distribution function (2.14) then becomes

$$
q_{v}(x)=-g^{2} \frac{\partial \bar{\Pi}(k, x)}{\partial k^{2}}=6 g^{2} \int \frac{d^{2} q_{\perp}}{(2 \pi)^{3}} \frac{\boldsymbol{q}_{\perp}^{2}+M^{2}}{\left(\boldsymbol{q}_{\perp}^{2}+M^{2}-m_{\pi}^{2} x(1-x)\right)^{2}},
$$

and since $1 / g^{2}=-\partial \bar{\Pi}(k) / \partial k^{2}\left(k^{2}=m_{\pi}^{2}\right)$ (see eq. (2.8) and (2.21)), the sum rules

$$
\int_{0}^{1} d x q_{v}(x)=1 ; \quad \int_{0}^{1} d x x q_{v}(x)=\frac{1}{2}
$$

follow immediately. The physical content of these relations is that we have one valence quark in the pion which carries half of the LC momentum $k_{-}$.

Finally, from the following identities

$$
-i \int \frac{d^{4} q}{(2 \pi)^{4}} \frac{1}{\left(q^{2}-\mu^{2}+i \epsilon\right)^{2}}=\frac{1}{2} \int d q_{-} \int \frac{d^{2} q_{\perp}}{(2 \pi)^{3}} \frac{\delta\left(q_{-}\right)}{\boldsymbol{q}_{\perp}^{2}+\mu^{2}}=\frac{1}{2} \int \frac{d^{2} q_{\perp}}{(2 \pi)^{3}} \frac{1}{\boldsymbol{q}_{\perp}^{2}+\mu^{2}}
$$

we note that: (i) One could rewrite the expression (2.26) for $q_{v}(x)$ in terms of a 4-dimensional integral, which agrees with the form given in previous works [8]. Such a procedure could be used in order to avoid the regularization in terms of LC coordinates, but we do not follow it in this paper. (ii) There is

\footnotetext{
${ }^{3}$ We define
}

$$
\int_{-\infty}^{\infty} \frac{d x}{x-a \pm i \epsilon} \equiv \lim _{R \rightarrow \infty} \int_{-R}^{R} \frac{d x}{x-a \pm i \epsilon}=\mp i \pi
$$

where the second equality follows from usual contour integration, taking into account the contribution from the semicircle. 
no contribution from the vertex correction shown in fig. 3: This graph, which arises from the structure of the constituent quark itself, gives a contribution $\propto \delta(x)$ when inserted into the graph of fig.2. Due to the regularization procedure discussed below, this gives no contribution.

\subsection{Regularization}

In the present ET formulation, we will regularize the gap equation (2.3), the bubble graph (2.6) and the expression for the pion decay constant (2.11) using the covariant 3-momentum cut-off $\left(\Lambda_{3}\right)$ as explained in ref. [22]. (This scheme will be denoted by '3M'.) Basically, this consists in integrating over $q_{0}$ using the theorem of residues, and then cutting the integral by $|\boldsymbol{q}|<\Lambda_{3}$. Since this procedure is not Lorentz invariant, however, for quantities with two-body intermediate states like (2.6) and (2.11) it is necessary to specify the frame where the integral is cut off, and then to 'boost' the result to a general frame. If we cut off the integral in the frame $\boldsymbol{k}=0$, the 'boost' for Lorentz scalar quantities simply means to replace $k_{0}^{2} \rightarrow k^{2}$ in the resulting expressions. In this way we obtain

$$
\begin{aligned}
1+2 \frac{G}{M}\langle\bar{\psi} \psi\rangle_{3 M}=1-24 G \int_{|\boldsymbol{q}|<\Lambda_{3}} \frac{d^{3} q}{(2 \pi)^{3}} \frac{1}{E_{q}} & =\frac{m}{M} \\
\Pi_{3 M}(k)=48 \int_{|\boldsymbol{q}|<\Lambda_{3}} \frac{d^{3} q}{(2 \pi)^{3}} \frac{E_{q}}{k^{2}-4 E_{q}^{2}+i \epsilon} & =\int_{4 M^{2}}^{\Lambda^{2}} d \mu^{2} \frac{\rho\left(\mu^{2}\right)}{k^{2}-\mu^{2}+i \epsilon} \\
f_{\pi}=-12 g M \int_{|\boldsymbol{q}|<\Lambda_{3}} \frac{d^{3} q}{(2 \pi)^{3}} \frac{1}{E_{q}\left(m_{\pi}^{2}-4 E_{q}^{2}\right)} & \equiv-g M I\left(m_{\pi}^{2}\right)
\end{aligned}
$$

where $E_{q}=\sqrt{M^{2}+\boldsymbol{q}^{2}}$, and the subscript $3 \mathrm{M}$ indicates that these quantities

refer to the 3-momentum cut-off procedure. In the second equality of eq. (2.30) we introduced $\mu^{2}=4\left(M^{2}+\boldsymbol{q}^{2}\right)$ to express $\Pi(k)$ as a dispersion integral 
with $\rho\left(\mu^{2}\right)=\frac{3 \mu^{2}}{4 \pi^{2}}\left(1-\frac{4 M^{2}}{\mu^{2}}\right)^{\frac{1}{2}}$, and $\Lambda^{2}=4\left(\Lambda_{3}^{2}+M^{2}\right)$.

The following important relation between the condensate and the bubble graph at $k=0$

$$
\langle\bar{\psi} \psi\rangle=M \Pi(0)
$$

which can be derived from the chiral Ward identity for the pion decay vertex (see Appendix B), is satisfied in the $3 \mathrm{M}$ regularization scheme, i.e; $\langle\bar{\psi} \psi\rangle_{3 M}=$ $M \Pi_{3 M}(0)$. Therefore the gap equation (2.29) and the pion pole condition $1+2 G \Pi\left(m_{\pi}^{2}\right)=0$ can be written as

$$
\begin{aligned}
1+2 G \Pi_{3 M}(0) & =\frac{m}{M} \\
\Pi_{3 M}\left(m_{\pi}^{2}\right)-\Pi_{3 M}(0) & =m_{\pi}^{2} I\left(m_{\pi}^{2}\right)=-\frac{m}{2 G M},
\end{aligned}
$$

where $I\left(k^{2}\right)$ was introduced in (2.31). Eq. (2.34) shows explicitly the Goldstone boson nature of the pion. The GOR relation is then obtained as follows:

$$
\begin{aligned}
-m\langle\bar{\psi} \psi\rangle_{3 M} & =\frac{M m}{2 G}\left(1-\frac{m}{M}\right)=-M^{2} m_{\pi}^{2} I\left(m_{\pi}^{2}\right)\left(1-\frac{m}{M}\right) \\
& =m_{\pi}^{2} f_{\pi}^{2}\left(1+m_{\pi}^{2} C\right)\left(1-\frac{m}{M}\right)
\end{aligned}
$$

with $C=\frac{1}{I\left(m_{\pi}^{2}\right)} \frac{\partial I\left(m_{\pi}^{2}\right)}{\partial m_{\pi}^{2}}$. In the last step we used the relation $f_{\pi}^{2}=-12 M^{2}$ $\times I\left(m_{\pi}^{2}\right) /\left(1+m_{\pi}^{2} C\right)$, which follows from (2.31) and $\frac{1}{g^{2}}=-\frac{\partial}{\partial k^{2}}\left(k^{2} I\left(k^{2}\right)\right)_{k^{2}=m_{\pi}^{2}}$.

For quantities expressed in terms of LC variables, the 'invariant mass cut-off' introduced by Lepage and Brodsky [21] is very convenient since it preserves all kinematic symmetries. (We will denote this cut-off scheme by 'LB'.) It is applicable to Feynman diagrams containing intermediate states with $n \geq 2$ particles propagating with respect to the LC 'time' variable $x^{+}$. For example, the bubble diagram $\bar{\Pi}(k, x)$ involves a $q \bar{q}$ intermediate state 
with invariant mass squared $2 k_{-}\left(e_{q}+e_{k-q}\right)=\left(\boldsymbol{q}_{\perp}^{2}+M^{2}\right) / x(1-x)$. (We set $\boldsymbol{k}_{\perp}=0$.) Requiring this to be smaller than $\Lambda^{2}$ leads to the 'LB condition'

$$
\mathrm{LB}: \quad \boldsymbol{q}_{\perp}^{2}<\Lambda^{2} x(1-x)-M^{2} \longrightarrow x_{1} \leq x \leq x_{2},
$$

where $x_{1,2}=\frac{1}{2}\left(1 \pm \sqrt{1-4 M^{2} / \Lambda^{2}}\right)$. This prescription regulates the ultraviolett as well as the infrared divergencies. The resulting regularized expressions $\bar{\Pi}_{L B}(k, x), \bar{\Pi}_{L B}(k)$ and $q_{v, L B}(x)$ are then given by eqs. (2.22), (2.23) and (2.26) with the condition (2.36).

A crucial question of course is whether this LB regularization procedure is consistent with the $3 \mathrm{M}$ procedure used above. For this, we note that if we perform a variable transformation $x \rightarrow q_{3}$ with

$$
x=\frac{1}{2}\left(1+\frac{q_{3}}{E_{q}}\right), \quad\left(E_{q}=\sqrt{\boldsymbol{q}_{\perp}^{2}+q_{3}^{2}+M^{2}}\right)
$$

in the expression for $\bar{\Pi}_{L B}(k)$, we exactly obtain $\Pi_{3 M}(k)$ of eq. (2.30) with $\Lambda^{2}=4\left(\Lambda_{3}^{2}+M^{2}\right)$. That is, the LB cut-off $\Lambda$ is the same as the dispersion cut-off $\Lambda$ introduced in (2.30). Similarly, if we evaluate the expression (2.11) for $f_{\pi}$ in terms of LC coordinates,

$$
f_{\pi}=6 g M \int d x \int \frac{d^{2} q_{\perp}}{(2 \pi)^{3}} \frac{1}{\boldsymbol{q}_{\perp}^{2}+M^{2}-m_{\pi}^{2} x(1-x)},
$$

impose the LB condition (2.36) and transform $x \rightarrow q_{3}$ as above, we arrive at the expression eq. (2.31). We therefore see that the $3 \mathrm{M}$ and LB cutoff procedures are consistent in the sense that (i) they lead to the same expression for $f_{\pi}$, and (ii) the quark distribution regularized with the LB cut-off involves the quantity $\bar{\Pi}_{L B}(k, x)$ (see eq. (2.26)), the $x$-integral over which coincides with the bubble graph $\Pi_{3 M}(k)$, i.e; $\bar{\Pi}_{L B}(k)=\Pi_{3 M}(k)$. 
At this point the question arises whether it is possible to consistently regularize all integrals in terms of the LC coordinates from the beginning, without making recourse to the regularization in terms of the usual coordinates. For this purpose, the LB prescription has to be extended, and this will be discussed in subsect. 3.5 .

\section{NJL model on the light cone}

In the ET quantization method used in the previous section, the introduction of LC variables is merely a change of variables. In this section we will investigate if we can obtain the same physical results in the framework of LC quantization. For this purpose, the main task is first to derive the form of the classical lagrangian in terms of the dynamical degrees of freedom, from which the hamiltonian and the canonical quantization conditions can be derived.

It is well known that in LC field theories not all components of the fermion field $\psi$ are independent degrees of freedom [11]. Namely, if we introduce projection operators

$$
\Lambda_{ \pm}=\frac{1}{2} \gamma^{\mp} \gamma^{ \pm}
$$

and split the fermion field according to $\psi=\Lambda_{+} \psi+\Lambda_{-} \psi \equiv \psi_{+}+\psi_{-}$, the component $\psi_{-}$is not a dynamical degree of freedom since there is no 'time' derivative $\partial_{+}$acting on it in the lagrangian (see eq. (3.4) below). Therefore, $\psi_{-}$should be eliminated by using the equation of motion before the theory is quantized. This can be easily done for theories which contain only fermionic bilinear terms, like free field theories or Yukawa theories [12]. For 4-fermi theories, however, the 'fermionic constraint equation' cannot be solved exactly $[19,18]$. 
In subsect. 3.1, we will use the $1 / N$ expansion to derive the effective LC lagrangian for $\psi_{+}$. Before plunging into the formalism, let us summarize here the result: The lagrangian is expressed in eq. (3.20), which shows that the interaction term has the form obtained by naively using the free field equations to eliminate $\psi_{-}$(see eqs. (3.9)), but there appears an effective 4 -fermi coupling constant $\bar{G}$ (see eq. (3.21) ) instead of the original $G$. In subsect. 3.2 we discuss the Feynman rules which follow from this effective lagrangian, and in subsects. 3.3 and 3.4 we re-derive the $\bar{q} q$ t-matrix in the pionic channel, the pion decay constant and the quark LC momentum distribution in the pion. There we will show that the results for these quantities are formally the same as obtained in the ET theory. In subsect. 3.5 we discuss two possible regularization procedures in terms of LC coordinates: An extended version of the LB scheme, and an alternative method [23] which avoids the introduction of a cut-off for the longitudinal momentum components.

\subsection{Effective LC lagrangian in the $1 / \mathrm{N}$ expansion}

To define the $1 / N$ expansion, we write as usual $G=\frac{G_{0}}{N}$ with $G_{0}$ independent of $N$. Let us first discuss the $\mathcal{O}(N)$ of the terms in the lagrangian (2.1). Splitting $\bar{\psi} \psi=<\bar{\psi} \psi>+(: \bar{\psi} \psi:)$, where $<\ldots>$ denotes a contraction with respect to the fermi fields, we have $<\bar{\psi} \psi>\propto N$ and $(: \bar{\psi} \psi:) \propto \sqrt{N}$. 团 Therefore the lagrangian (2.1) contains a c-number part of $\mathcal{O}(N)$, a 1-body part of order $\mathcal{O}(\sqrt{N})$, and a 2-body part of order $\mathcal{O}(1)$. Our aim here is to give a similar decomposition of the lagrangian in the LC theory, i.e; to derive the 1-body part to $\mathcal{O}(\sqrt{N})$ and the 2-body part to $\mathcal{O}(1)$.

\footnotetext{
${ }^{4}$ This follows from $<(: \bar{\psi} \psi:)^{2}>\propto N$. A similar consideration shows that in the decomposition $(: \bar{\psi} \psi:)^{2}=<(: \bar{\psi} \psi:)^{2}>+\left(:(: \bar{\psi} \psi:)^{2}:\right)$ both terms are of $\mathcal{O}(N)$.
} 
To formulate the $1 / N$ expansion in the LC theory, it is convenient to introduce bosonic auxiliary fields $\sigma$ and $\boldsymbol{\pi}$. The lagrangian (2.1) is equivalent to

$$
\mathcal{L}=\bar{\psi}(\not \not \supset-\hat{M}) \psi-\frac{G_{0}}{N}\left(\sigma^{2}+\boldsymbol{\pi}^{2}\right),
$$

where

$$
\hat{M}=m+2 \frac{G_{0}}{N}\left(\sigma+i \boldsymbol{\pi} \cdot \boldsymbol{\tau} \gamma_{5}\right) .
$$

We then rewrite this lagrangian in terms of $\mathrm{LC}$ variables, making use of the projection operators (3.1). Some useful relations for quark bilinears are given in Appendix A, and we obtain

$$
\begin{aligned}
\mathcal{L} & =\sqrt{2}\left(\psi_{+}^{\dagger} i \partial_{+} \psi_{+}+\psi_{-}^{\dagger} i \partial_{-} \psi_{-}\right)-\frac{G_{0}}{N}\left(\sigma^{2}+\boldsymbol{\pi}^{2}\right) \\
& -\psi_{+}^{\dagger}\left(i \boldsymbol{\alpha}_{\perp} \cdot \boldsymbol{\partial}_{\perp}+\gamma^{0} \hat{M}\right) \psi_{-}-\psi_{-}^{\dagger}\left(i \boldsymbol{\alpha}_{\perp} \cdot \boldsymbol{\partial}_{\perp}+\gamma^{0} \hat{M}\right) \psi_{+} .
\end{aligned}
$$

Since we introduced the bosonic auxiliary fields, the fermionic constraint equation $\partial \mathcal{L} / \partial \psi_{-}^{\dagger}=0$ can be solved explicitly: ๒

$$
\psi_{-}=\frac{1}{\sqrt{2} i \partial_{-}}\left(i \boldsymbol{\alpha}_{\perp} \cdot \boldsymbol{\partial}_{\perp}+\gamma^{0} \hat{M}\right) \psi_{+},
$$

and inserting this into eq. (3.4) we obtain

$$
\begin{aligned}
\mathcal{L} & =\sqrt{2} \psi_{+}^{\dagger} i \partial_{+} \psi_{+}-\frac{G_{0}}{N}\left(\sigma^{2}+\boldsymbol{\pi}^{2}\right) \\
& -\frac{1}{\sqrt{2}} \psi_{+}^{\dagger}\left(i \boldsymbol{\alpha}_{\perp} \cdot \boldsymbol{\partial}_{\perp}+\gamma^{0} \hat{M}\right) \frac{1}{i \partial_{-}}\left(i \boldsymbol{\alpha}_{\perp} \cdot \boldsymbol{\partial}_{\perp}+\gamma^{0} \hat{M}\right) \psi_{+} .
\end{aligned}
$$

${ }^{5}$ We use the following definition of $\frac{1}{\partial_{-}}$:

$$
\left(\frac{1}{\partial_{-}} f\right)\left(x^{-}\right) \equiv \frac{1}{2} \int_{-\infty}^{\infty} d y^{-} \epsilon\left(x^{-}-y^{-}\right) f\left(y^{-}\right)
$$

with $\epsilon(x)=\Theta(x)-\Theta(-x)$. 
We now separate the leading order $(N)$ of the 'c-number part' of the $\sigma$ field by writing $\sigma=\sigma_{0} N+\hat{\sigma}$. Here $\hat{\sigma}$ contains the leading order $(\sqrt{N})$ of the 'fluctuation part', as well as the next-to-leading order $\left(N^{0}\right)$ of both the c-number and the fluctuation part. From the form of the lagrangian (3.6) it is clear that up to the order which concerns us here (that is, $\mathcal{O}(\sqrt{N})$ for the 1-body part, $\mathcal{O}\left(N^{0}\right)$ for the 2-body part) it is sufficient to consider $\hat{\sigma}$ (and also $\boldsymbol{\pi})$ as the fluctuation piece of $\mathcal{O}(\sqrt{N})$. We define the constituent quark mass as

$$
M=m+2 G_{0} \sigma_{0} .
$$

To split the lagrangian into 'free' and 'interaction' parts, it is convenient to follow ref. [12 and define the fermion field

$$
\Psi \equiv \Psi_{+}+\Psi_{-}
$$

with

$$
\Psi_{+} \equiv \psi_{+} ; \quad \Psi_{-} \equiv \frac{1}{\sqrt{2} i \partial_{-}}\left(i \boldsymbol{\alpha}_{\perp} \cdot \boldsymbol{\partial}_{\perp}+\gamma^{0} M\right) \psi_{+} \cdot
$$

In terms of this field the lagrangian (3.6) can be written as

$$
\begin{aligned}
\mathcal{L} & =\mathcal{L}_{0}-2 \frac{G_{0}}{N} \bar{\Psi}\left(\hat{\sigma}+i \boldsymbol{\pi} \cdot \boldsymbol{\tau} \gamma_{5}\right) \Psi-\frac{G_{0}}{N}\left(\hat{\sigma}^{2}+\boldsymbol{\pi}^{2}\right)-2 G_{0} \sigma_{0} \hat{\sigma} \\
& -2\left(\frac{G_{0}}{N}\right)^{2} \bar{\Psi}\left(\hat{\sigma}+i \boldsymbol{\pi} \cdot \boldsymbol{\tau} \gamma_{5}\right) \gamma^{+} \frac{1}{i \partial_{-}}\left(\hat{\sigma}+i \boldsymbol{\pi} \cdot \boldsymbol{\tau} \gamma_{5}\right) \Psi
\end{aligned}
$$

with

$$
\begin{aligned}
\mathcal{L}_{0} & =\bar{\Psi}(i \not \partial-M) \Psi \\
& =\sqrt{2} \psi_{+}^{\dagger} i \partial_{+} \psi_{+}-\frac{1}{\sqrt{2}} \psi_{+}^{\dagger}\left(i \boldsymbol{\alpha}_{\perp} \cdot \boldsymbol{\partial}_{\perp}+\gamma^{0} M\right) \frac{1}{i \partial_{-}}\left(i \boldsymbol{\alpha}_{\perp} \cdot \boldsymbol{\partial}_{\perp}+\gamma^{0} M\right) \psi_{+}
\end{aligned}
$$


We note that up to the required order it is sufficient to replace the two fermi fields in the last term of (3.10) by a contraction. To exhibit the dependence of the lagrangian on the bosonic auxiliary fields more explicitly, we express it in terms of the combined field $\Phi \equiv(\hat{\sigma}, \boldsymbol{\pi})\left(\right.$ i.e; $\left.\Phi^{(0)}=\hat{\sigma}, \Phi^{(i)}=\pi^{i}\right)$ and the corresponding vertex operator $\Gamma \equiv\left(1, i \gamma_{5} \boldsymbol{\tau}\right)$ as

$$
\mathcal{L}=\mathcal{L}_{0}-\frac{1}{2} \Phi^{(\alpha)} \vec{A}_{\alpha \beta} \Phi^{(\beta)}-\Phi^{(\alpha)} B_{\alpha}
$$

where we introduced

$$
\begin{aligned}
\vec{A}_{\alpha \beta} & =2 \frac{G_{0}}{N} \delta_{\alpha \beta}+\left(2 \frac{G_{0}}{N}\right)^{2}\left\langle\bar{\Psi} \Gamma_{\alpha} \gamma^{+} \frac{1}{i \vec{\partial}_{-}} \Gamma_{\beta} \Psi\right\rangle \\
B_{\alpha} & =2 \delta_{\alpha 0} G_{0} \sigma_{0}+2 \frac{G_{0}}{N} \bar{\Psi} \Gamma_{\alpha} \Psi .
\end{aligned}
$$

We now eliminate the auxiliary boson fields using $\partial \mathcal{L} / \partial \Phi=-A \Phi-B=0$, which gives formally

$$
\Phi=-A^{-1} B ; \quad \mathcal{L}=\mathcal{L}_{0}+\frac{1}{2} B^{T} A^{-1} B
$$

The matrix operator $A$ introduced above is defined by 0

$$
A_{\alpha \beta} \Phi^{(\beta)} \equiv \frac{1}{2}\left(\vec{A}_{\alpha \beta} \Phi^{(\beta)}+\Phi^{(\beta)} \overleftarrow{A}_{\beta \alpha}\right)
$$

Due to parity and/or isospin invariance, $A_{\alpha \beta} \propto \delta_{\alpha \beta}$, and we therefore define

$$
A_{\alpha \beta} \equiv \delta_{\alpha \beta} 2 \frac{G_{0}}{N}\left(1+2 \frac{G_{0}}{N} F\right)
$$

\footnotetext{
${ }^{6}$ The quantity $\overleftarrow{A}$ involves $\frac{1}{\vec{\partial}_{-}}=\frac{1}{\partial_{-}}$acting to the right, while $\overleftarrow{A}$ involves $\frac{-1}{\partial_{-}}$acting to the left. The symmetrized form of $A$ in $(3.16)$ appears naturally if we take the functional derivative of the action $\int d^{4} x \mathcal{L}(x)$ with respect to $\Phi(x)$, using the definition of $1 / \partial_{-}$. We note that also in the lagrangian eq. (3.12), $\vec{A}_{\alpha \beta}$ can be replaced by $A_{\alpha \beta}$, since this leaves the action unchanged.
} 
where the quantity

$$
F=\frac{1}{2}\left(<\bar{\Psi} \gamma^{+} \frac{1}{i \vec{\partial}_{-}} \Psi>+<\bar{\Psi} \gamma^{+} \frac{-1}{i \overleftarrow{\partial}_{-}} \Psi>\right)
$$

is of $\mathcal{O}(N)$.

$\sigma_{0}$ is determined by the requirement $\langle\Phi\rangle=-A^{-1}\langle B\rangle \equiv 0$. Due to (3.14) and (3.7), this requirement $\langle B\rangle=0$ leads to the gap equation

$$
M=m-2 G<\bar{\Psi} \Psi>
$$

Since in this leading order $(N)$ we have $<\bar{\Psi} \Psi>=<\bar{\psi} \psi>$, this is formally the same gap equation as obtained in the ET theory, eq. (2.3).

Since $\langle B\rangle=0$, the interaction term in the lagrangian (3.15) becomes $\frac{1}{2}(: B:) A^{-1}(: B:)$. We therefore arrive at the final form of the LC lagrangian: \

$$
\mathcal{L}=\mathcal{L}_{0}+\bar{G}\left((: \bar{\Psi} \Psi:)^{2}-\left(: \bar{\Psi} \gamma_{5} \tau \Psi:\right)^{2}\right)
$$

where $\mathcal{L}_{0}$ of eq. (3.11) contains the 1-body part of $\mathcal{O}(\sqrt{N})$, and the 2-body part is of order $N^{0}$ and involves the effective coupling constant

$$
\bar{G}=\frac{G}{1+2 G F}
$$

with $G=G_{0} / N$. One should note that the lagrangian (3.20) contains only $\psi_{+}$as a dynamical component, since we defined the field $\Psi$ in terms of $\psi_{+}$ in eqs. (3.8), (3.9). Our result is that the effective interaction lagrangian in the LC theory up to $\mathcal{O}\left(N^{0}\right)$ can be obtained from eq. 2.1 by using the free field equations to eliminate $\psi_{-}$, but the coupling constant $G$ is replaced

\footnotetext{
${ }^{7}$ Our notation used in eq. $(\sqrt{3.20})$ anticipates the fact derived in the next subsection that in coordinate space the quantity $\bar{G} \propto \delta\left(x^{-}-y^{-}\right)$, i.e; that the interaction lagrangian is local although $F$ of eq. (3.18) is apparently non-local.
} 
by the effective coupling constant $\bar{G}$ of eq. (3.21). (The explicit expression for the quantity $F$ will be given in eq. (3.34) below.)

Once we have derived the classical lagrangian for $\psi_{+}$, the quantization proceeds as usual by imposing anti-commutation relations between $\psi_{+}$and $\Pi_{+}=\sqrt{2} i \psi_{+}^{\dagger}$ at equal LC times $x^{+}$. The mode expansion of the free field reads 8

$$
\psi_{+}(x)=\int \frac{d^{3} p}{(2 \pi)^{3 / 2}} \frac{1}{\sqrt{2 p_{-}}} \sum_{s}\left[b_{\boldsymbol{p}, s} u_{+}(\boldsymbol{p}, s) e^{-i \bar{p} \cdot x}+d_{\boldsymbol{p}, s}^{\dagger} v_{+}(\boldsymbol{p}, s) e^{i \bar{p} \cdot x}\right]
$$

with $d^{3} p=d p_{-} d^{2} p_{\perp}, \boldsymbol{p}=\left(p_{-}, \boldsymbol{p}_{\perp}\right), p \cdot x \equiv p_{+} x^{+}+p_{-} x^{-}-\boldsymbol{p}_{\perp} \cdot \boldsymbol{x}_{\perp}$, and $\bar{p}^{\mu}$ was defined below (2.20). Using the mode expansion given above, one obtains for the free fermion propagator

$$
\begin{aligned}
\bar{S}_{++}(p) & \equiv F \cdot T \cdot\left(-i<0\left|\tilde{T}\left(\psi_{+} \psi_{+}^{\dagger}\right)\right| 0>\right) \\
& =\frac{\Lambda_{+} \sqrt{2} p_{-}}{p^{2}-M^{2}+i \epsilon},
\end{aligned}
$$

where $\tilde{T}$ orders the operators with respect to $x^{+}$. The quantity $1 /\left(p^{2}-M^{2}+\right.$ $i \epsilon)$ has been given in terms of $\mathrm{LC}$ variables in eq. (2.18).

\subsection{Feynman rules, gap equation and effective cou- pling constant}

To calculate a fermionic Green function

$$
<0\left|\tilde{T}\left(\psi_{+}\left(x_{1}\right) \ldots \psi_{+}^{\dagger}\left(x_{n}\right)\right)\right| 0>
$$

one could formulate the Feynman rules directly in terms of the field $\psi_{+}$, using the lagrangian (3.20) and the definition of $\Psi$ in terms of $\psi_{+}$given in

\footnotetext{
${ }^{8}$ Properties of the LC spinors $u_{ \pm}=\Lambda_{ \pm} u$ and $v_{ \pm}=\Lambda_{ \pm} v$ are summarized in Appendix
} A. 
eq. (3.8). In this method, one uses the propagator eq. (3.23) for internal fermion lines, and 4-fermi vertices of the following form, referring e.g; to the pionic $q \bar{q}$ channel,

$$
\begin{aligned}
& -2 i \bar{G} X_{k}^{i}\left(\boldsymbol{q}^{\prime}\right) X_{k}^{i}(\boldsymbol{q}) \\
& \equiv-2 i \bar{G}\left[\gamma_{5} \gamma^{0} \tau_{i}\left(Z\left(\boldsymbol{q}^{\prime}\right)-Z\left(\boldsymbol{q}^{\prime}-\boldsymbol{k}\right)\right)\right]\left[\gamma_{5} \gamma^{0} \tau_{i}(Z(\boldsymbol{q}-\boldsymbol{k})-Z(\boldsymbol{q}))\right]
\end{aligned}
$$

where the external momenta are assigned as in fig. 1, and

$$
Z(\boldsymbol{p})=\left(\boldsymbol{\alpha}_{\perp} \cdot \boldsymbol{p}_{\perp}+\gamma^{0} M\right) \frac{1}{\sqrt{2} p_{-}} .
$$

A much more convenient method, however, is first to calculate the Green function

$$
<0\left|\tilde{T}\left(\Psi\left(x_{1}\right) \ldots \Psi^{\dagger}\left(x_{n}\right)\right)\right| 0>
$$

and then to project out (3.24) by applying the projection operator $\Lambda_{+}$to each external fermion leg. In this method, which will be used in the next section to obtain the $q \bar{q}$ t-matrix in the pionic channel, one uses the propagator

$$
\bar{S}(p) \equiv F . T .\left(-i<0\left|\tilde{T}\left(\Psi \Psi^{\dagger}\right)\right| 0>\right)=\frac{\bar{p}+M}{p^{2}-M^{2}+i \epsilon} \gamma^{0}=S(p)-\frac{\gamma^{+} \gamma^{0}}{2 p_{-}}
$$

for each internal fermion line, and (constant) 4-fermi vertices which differ from those in the ET treatment of the NJL model only by the replacement $G \rightarrow \bar{G}$. The form of $\bar{S}(p)$ given in eq. (3.28) can be easily derived as follows [12]: Splitting $\bar{S}(p)$ into the four components $\bar{S}_{i j}(i, j=+,-)$, where $\bar{S}_{++}$ is given by eq. (3.23), we have, using the form of $\Psi$ as given by eq.(3.8) and 
the Feynman propagator (2.17) (see also Appendix A):

$$
\begin{aligned}
& \bar{S}_{+-}(p)=\bar{S}_{++}(p) Z(\boldsymbol{p})=\Lambda_{+} S(p) \Lambda_{-} \\
& \bar{S}_{-+}(p)=Z(\boldsymbol{p}) \bar{S}_{++}(p)=\Lambda_{-} S(p) \Lambda_{+} \\
& \bar{S}_{--}(p)=Z(\boldsymbol{p}) \bar{S}_{++}(p) Z(\boldsymbol{p})=\Lambda_{-} S(p) \Lambda_{-}-\frac{\Lambda_{-}}{\sqrt{2} p_{-}} .
\end{aligned}
$$

¿From these relations one easily obtains eq. (3.28). This propagator $\bar{S}(p)$ has been introduced already in eq. (2.20) by separating the contact term from the Feynman propagator $S(p)$.

Clearly, if the ET and the LC treatments of the NJL model are equivalent, the contributions due to additional term $-\gamma^{+} \gamma^{0} / 2 p_{-}$in the propagator and the modifications due to the replacement $G \rightarrow \bar{G}$ in the vertices should 'cancel' for physical observables. These are the same kinds of cancellations as observed for the $\sigma$-model in ref. [12].

Using the propagator (3.28), it is now easy to derive the explicit forms of the gap equation and the quantity $\mathrm{F}$, which determines the effective coupling constant (see eqs. (3.18) and (3.21)). For the gap equation we obtain from (3.7) and (3.19)

$$
M=m-2 G\langle\bar{\Psi} \Psi\rangle=m+12 i G \int \frac{d^{4} q}{(2 \pi)^{4}} \operatorname{tr}_{D}\left(\bar{S}(q) \gamma^{0}\right),
$$

and since from eq. (3.28) we have $\operatorname{tr}_{D}\left(\bar{S}(q) \gamma^{0}\right)=\operatorname{tr}_{D}\left(S(q) \gamma^{0}\right)$, we see that the gap equation is formally the same as in the ET theory. Using (2.18) and performing the $q_{+}$integral we obtain

$$
M-m=48 i G M \int \frac{d^{4} q}{(2 \pi)^{4}} \frac{1}{q^{2}-M^{2}+i \epsilon}=24 G M \int_{0}^{\infty} \frac{d q_{-}}{q_{-}} \int \frac{d^{2} q_{\perp}}{(2 \pi)^{3}} .
$$

Although it seems from this expression that in the case $m=0$ only the trivial solution $(M=0)$ exists, we have to note that the theory has not yet been regularized. (See subsect. 3.5.) 
Let us now turn to the quantity $F$ of eq.(3.18). Using the definition of $\frac{1}{\partial_{-}}$given earlier, we have for the quantity $A_{\alpha \beta} \equiv \delta_{\alpha \beta} a$ of eq.(3.17)

$$
a\left(z^{-}\right)=2 G\left[\delta\left(z^{-}\right)+3 G \epsilon\left(z^{-}\right) \operatorname{tr}_{D}\left(\bar{S}\left(z^{-}\right) \gamma^{0} \gamma^{+}-\bar{S}\left(-z^{-}\right) \gamma^{0} \gamma^{+}\right)\right]
$$

where $z^{-}=x^{-}-y^{-}$. The Fourier transform of this is

$$
a\left(k_{-}\right)=2 G\left(1-6 i G \int \frac{d^{4} q}{(2 \pi)^{4}} t_{D}\left(\bar{S}(q) \gamma^{0} \gamma^{+}\right)\left(\frac{\mathcal{P}}{q_{-}+k_{-}}+\frac{\mathcal{P}}{q_{-}-k_{-}}\right)\right) .
$$

Again we note that in this expression $\bar{S}(p)$ can be replaced by the Feynman propagator $S(p)$ without any modification. Performing the $q_{+}$integral using (2.18) and introducing $q_{-}=k_{-} x$ we see that $a$ (or $F$ ) is actually a constant, and so is $\bar{G}$ of eq.(3.21). The expression for $F$ becomes

$$
F=-6 \mathcal{P} \int_{0}^{\infty} d x\left(\frac{1}{x+1}+\frac{1}{x-1}\right) \int \frac{d^{2} q_{\perp}}{(2 \pi)^{3}} .
$$

\subsection{Pionic t-matrix}

According to the discussion at the beginning of the previous subsection, to obtain the $q \bar{q}$ t-matrix in the $\mathrm{LC}$ formulation, we first determine the t-matrix as-

sociated with the amputated connected Green function $<0\left|\tilde{T}\left(\Psi \Psi \Psi^{\dagger} \Psi^{\dagger}\right)\right| 0>$. In the ladder approximation this is obtained by summing the bubble graphs $\bar{\Pi}(k)$, which have been introduced already in sect. 2 , in the same way as in the ET theory as

$$
\bar{t}(k)=\left(\gamma^{0} \gamma_{5} \tau_{i}\right) \bar{\tau}(k)\left(\gamma^{0} \gamma_{5} \tau_{i}\right)
$$

with

$$
\begin{aligned}
\bar{\tau}(k) & =\frac{-2 i \bar{G}}{1+2 \bar{G} \bar{\Pi}(k)} \\
\bar{\Pi}(k) & =6 i \int \frac{d^{4} q}{(2 \pi)^{4}} \operatorname{tr}_{D}\left(\gamma^{0} \gamma_{5} \bar{S}(q) \gamma^{0} \gamma_{5} \bar{S}(q-k)\right) .
\end{aligned}
$$


The t-matrix associated with the amputated connected Green function $<0\left|\tilde{T}\left(\psi_{+} \psi_{+} \psi_{+}^{\dagger} \psi_{+}^{\dagger}\right)\right| 0>$ is then simply obtained by projection (see fig. 1 for the assignment of the external momenta):

$$
\begin{aligned}
& \bar{S}_{++}\left(q^{\prime}\right) \bar{S}_{++}\left(q^{\prime}-k\right) \tilde{t} \bar{S}_{++}(q-k) \bar{S}_{++}(q) \\
= & \Lambda_{+}\left(\bar{S}\left(q^{\prime}\right) \gamma^{0} \gamma_{5} \tau_{i} \bar{S}\left(q^{\prime}-k\right)\right) \Lambda_{+} \bar{\tau}(k) \Lambda_{+}\left(\bar{S}(q-k) \gamma^{0} \gamma_{5} \tau_{i} \bar{S}(q)\right) \Lambda_{+}
\end{aligned}
$$

The result of this projection is $\tilde{t}=X_{k}^{i}\left(\boldsymbol{q}^{\prime}\right) \bar{\tau}(k) X_{k}^{i}(\boldsymbol{q})$, i.e; the vertices $\left(\gamma^{0} \gamma_{5} \tau_{i}\right)$ are replaced by the momentum dependent vertices $X_{k}^{i}$ of eq.(3.25). Of course, the pole structure is not affected by this projection.

Using eq.(3.21), we can rewrite the reduced t-matrix (3.36) as

$$
\bar{\tau}(k)=\frac{-2 i G}{1+2 G(\bar{\Pi}(k)+F)}
$$

Comparing with eq. (2.5), we see that we should have the relation

$$
\Pi(k)=\bar{\Pi}(k)+F
$$

with $F$ given by (3.34), in order that the reduced t-matrices, and in particular the pole parts, in ET and LC theories are the same. On the other hand, by explicit evaluation we found that $\Pi(k)=\bar{\Pi}(k)+R$ (see eq.(2.21)), where $R$ is given by (2.25). Formally, the relation

$$
F=R
$$

holds, but to establish it from (2.25) and (3.34) one has to introduce variable shifts in divergent integrals.

The Goldstone nature of the pion in the present LC treatment follows by noting that the relation $(2.32)$, which now reads

$$
<\bar{\Psi} \Psi>=M(\bar{\Pi}(0)+R),
$$


is formally satisfied. The explicit verification by using (3.31), (2.22) and (2.25), however, again involves variable shifts in divergent integrals. If one nevertheless assumes the validity of (3.42) and (3.41), one can write the gap equation (3.30) and the pion pole condition $1+2 \bar{G} \bar{\Pi}\left(m_{\pi}^{2}\right)=0$ as follows:

$$
\begin{aligned}
1+2 \bar{G} \bar{\Pi}(0) & =\frac{\bar{m}}{M} \\
\bar{\Pi}\left(m_{\pi}^{2}\right)-\bar{\Pi}(0) & =m_{\pi}^{2} I\left(m_{\pi}^{2}\right)=-\frac{\bar{m}}{2 \bar{G} M},
\end{aligned}
$$

where $I\left(k^{2}\right)$ was introduced in (2.31). Here we used (3.21) and (2.32) to express the gap equation in terms of $\bar{G}$ and $\bar{\Pi}(0)$, and introduced

$$
\bar{m}=\frac{m}{1+2 G F}=m \frac{\bar{G}}{G} .
$$

Eq. (3.44) shows the Goldstone nature of the pion. We note the the GOR relation is satisfied with the current quark mass $m$ (not $\bar{m}$ ). Its derivation follows the same steps as outlined in eq. (2.35).

\subsection{Bound state matrix elements and pionic LC wave function}

In contrast to the BS wave function introduced in sect.2, a 'LC wave function' usually refers to the solution of a Schrödinger-type (or, more precisely, TDAtype) equation which can be derived directly in the hamiltonian formalism (see Appendix C). Here we want to show how this kind of wave function can also be obtained by using the projection methods introduced in the previous sections.

In the same way as in the ET theory, we first define a BS wave function $\bar{\Phi}$ in the LC theory by

$$
\bar{S}\left(q^{\prime}\right) \bar{S}\left(q^{\prime}-k\right) \bar{t} \bar{S}(q-k) \bar{S}(q) \rightarrow \frac{i}{2 k_{-}} \frac{\bar{\Phi}_{k}^{i}\left(q^{\prime}\right) \bar{\Phi}_{k}^{i \dagger}(q)}{k_{+}-e_{k}+i \epsilon} \quad \text { as } k_{+} \rightarrow e_{k} .
$$


This wave function $\bar{\Phi}_{k}(q)$ is given by (2.8) with $S \rightarrow \bar{S}$. The BS wave function for the dynamical $(+)$ fermion components $(\tilde{\Phi})$ is defined by (see eq. (3.38))

$$
\bar{S}_{++}\left(q^{\prime}\right) \bar{S}_{++}\left(q^{\prime}-k\right) \tilde{t} \bar{S}_{++}(q-k) \bar{S}_{++}(q) \rightarrow \frac{i}{2 k_{-}} \frac{\tilde{\Phi}_{k}^{i}\left(q^{\prime}\right) \tilde{\Phi}_{k}^{i \dagger}(q)}{k_{+}-e_{k}+i \epsilon} \quad \text { as } k_{+} \rightarrow e_{k} .
$$

It is clear that

$$
\tilde{\Phi}_{k}^{i}(q)=\Lambda_{+} \bar{\Phi}_{k}(q) \Lambda_{+}=g\left(\Lambda_{+} \bar{S}(q) \gamma^{0} \gamma_{5} \tau_{i} \bar{S}(q-k) \Lambda_{+}\right)
$$

Due to $\Lambda_{+} \gamma^{+}=\gamma^{+} \gamma^{0} \Lambda_{+}=0$ and (3.28), the propagator $\bar{S}$ can be replaced by the Feynman propagator $S$ in this expression, and $\tilde{\Phi}$ agrees with the ++ component of the BS wave function (2.8).

The calculation of bound state matrix elements in the LC theory can then proceed by using these BS wave functions in the same way as in the ET theory. As in the case of the Green functions, one has two choices: One is to use the wave function $\tilde{\Phi}$ together with the vertices and propagators defined in terms of the field $\psi_{+}$, and the other, more convenient method is to use the wave function $\bar{\Phi}$ together with the vertices and propagators defined for the field $\Psi$. In Appendix D it is shown that the calculation of the pion decay constant and the valence quark distribution in the pion along these lines leads to the same results as in the ET theory, namely eq.(2.11) and eq. (2.14).

Another method to calculate matrix elements involving bound state like those in (2.9) and (2.12), which is more specific to LC theories, is to use the Fock space expansion of the pion state $\mid k i>$. Let us explain this approach here in order to make contact to the concept of Schrödinger-type LC wave functions. It is well known 25] that, for interactions which do not depend on 
the 'time' component of the relative momentum ( $q_{+}$here), the Schrödingertype (equal 'time') wave function is obtained by integrating the BS wave function over $q_{+}$:

$$
\begin{aligned}
& \Phi_{k}^{i(S)}(\boldsymbol{q})=\int \frac{d q_{+}}{2 \pi i} \tilde{\Phi}_{k}^{i}(q) \\
= & g \int \frac{d q_{+}}{2 \pi i}\left(\Lambda_{+} \bar{S}(q) \gamma^{0} \gamma^{5} \tau_{i} \bar{S}(q-k) \Lambda_{+}\right) .
\end{aligned}
$$

As shown in Appendix C, this 'LC wave function' satisfies the same Schrödingertype equation as derived directly in the hamiltonian formalism. If we use the propagator (3.28) with the pole part given in eq.(2.18) in this expression and consider the case $k_{-}>0$, we get the restrictions $q_{-}>0$ and $q_{-}-k_{-}<0$, and therefore the LC wave function (3.49) is non-zero only for $0<x<1$, where $x=q_{-} / k_{-}$. To go from the Dirac to the spin representation, we multiply eq.(3.49) from left by $\left(\sqrt{2} q_{-}\right)^{-1 / 2} u_{+}^{\dagger}\left(\boldsymbol{q}, s_{1}\right)$ and from right by $\left(\sqrt{2}\left(k_{-}-q_{-}\right)\right)^{-1 / 2} v_{+}\left(\boldsymbol{k}-\boldsymbol{q}, s_{2}\right)$. Multiplying also the corresponding isospin $(t)$ and color $(c)$ wave functions, and denoting $a \equiv(s, t, c)$, we obtain after a simple calculation

$$
\begin{aligned}
\Phi_{k, a_{1} a_{2}}^{i(S)}(\boldsymbol{q}) & =\frac{g}{2 k_{-}} \frac{1}{\sqrt{x(1-x)}} \frac{1}{m_{\pi}^{2} x(1-x)-\boldsymbol{q}_{\perp}^{2}-M^{2}}\left(\chi_{t_{1}}^{\dagger} \tau_{i} \chi_{t_{2}}\right) \delta_{c 1, c 2} \\
& \times u_{+}^{\dagger}\left(\boldsymbol{q}, s_{1}\right) \gamma_{5}\left(\boldsymbol{\gamma}_{\perp} \cdot \boldsymbol{q}_{\perp}+M\right) v_{+}\left(\boldsymbol{k}-\boldsymbol{q}, s_{2}\right) .
\end{aligned}
$$

The conventional normalization

$$
\int \frac{d^{2} q_{\perp}}{(2 \pi)^{3}} \int_{0}^{1} d x \sum_{a_{1} a_{2}}\left|\Phi_{k, a_{1} a_{2}}^{i(S)}\left(q_{-}=k_{-} x, \boldsymbol{q}_{\perp}\right)\right|^{2}=1
$$

can be checked from eq. (3.50), using $1 / g=\left[-\partial \bar{\Pi} / \partial k^{2}\right]^{\frac{1}{2}}\left(k^{2}=m_{\pi}^{2}\right)$ with $\bar{\Pi}(k)$ given by (2.23). The Fock space expansion of the pionic bound state in 
the TDA is then given by

$$
\left|k i>=\sqrt{2} \int_{0}^{k_{-}} d q_{-} \int d^{2} q_{\perp} \sum_{a_{1} a_{2}} \Phi_{k, a_{1} a_{2}}^{i(S)}(\boldsymbol{q}) b_{\boldsymbol{q}, a_{1}}^{\dagger} d_{\boldsymbol{k}-\boldsymbol{q}, a_{2}}^{\dagger}\right| 0>.
$$

If we note that $\bar{\psi}(0) \gamma^{+} \psi\left(z^{-}\right)=\sqrt{2} \psi_{+}^{\dagger}(0) \psi_{+}\left(z^{-}\right)$in eq.(2.12), we can use the mode expansion (3.22) (supplemented by the isospin and color parts) and the Fock expansion (3.52) to obtain after a simple calculation

$$
q_{v}(x)=\int \frac{d^{2} q_{\perp}}{(2 \pi)^{3}} \sum_{a_{1} a_{2}}\left|\Phi_{k, a_{1} a_{2}}^{i(S)}\left(q_{-}=k_{-} x, \boldsymbol{q}_{\perp}\right)\right|^{2} .
$$

The evaluation of the sum then leads to the same result as obtained in the ET theory, namely eq.(2.26).

In a similar way, by noting that the + component of the axial vector

current is given by $\frac{1}{\sqrt{2}} \psi_{+}^{\dagger} \gamma_{5} \boldsymbol{\tau} \psi_{+}$, we obtain from eq. (2.9) the expression (2.38) (yet unregularized).

\subsection{Regularization schemes in LC theory}

In the previous subsections we have shown that the NJL model on the LC gives formally the same results for the pion decay constant, the gap equation, the pion pole condition and the quark distribution in the pion as the ET theory. In sect. 2 we noted that the bubble graphs in the LC theory (like $\bar{\Pi}(k, x)$ and $\bar{\Pi}(k))$ can be regularized in a covariant way consistently with the $3 \mathrm{M}$ regularization scheme in the ET theory. To give consistent regularization prescriptions for tadpole graphs in the LC theory, like the quark condensate or the quantity $F$ of eq. (3.34), turns out to be a very difficult task which we do not attempt to solve explicitly in this work. We will instead make use of the general relation (3.42). We have shown that this relation holds formally in the LC theory, if one performs variable shifts in divergent longitudinal 
momentum $\left(q_{-}\right)$integrals. Here we will assume that (3.42) and also (3.41) are valid even in the regularized theory. This method of defining the quark condensate in LC theories in terms of correlation functions has been used in several recent works [26].

Following this method, we will give two possible regularization schemes below. The first one ('extended LB scheme') is equivalent to the 3M cut-off scheme in the ET formulation. The second one ('transverse cut-off scheme'), which was proposed in ref. 23], avoids the introduction of a cut-off for the longitudinal momentum component.

\subsubsection{Extended LB scheme}

In addition to the LB condition (2.36) for the 2-point functions, in the 'extended LB scheme' one assumes the validity of eqs. (3.42) and (3.41). Then the gap equation and the pion pole condition assume the forms (3.43) and (3.44) with $\bar{\Pi} \rightarrow \bar{\Pi}_{L B}$. In particular, the gap equation takes the explicit form

$$
1-24 \bar{G} \int_{L B} \frac{d x}{x} \int_{L B} \frac{d^{2} q_{\perp}}{(2 \pi)^{3}}=\frac{\bar{m}}{M},
$$

where the LB condition is given by (2.36). Performing the variable transformation (2.37), we see that this is just the gap equation in the $3 \mathrm{M}$ regularization scheme (eq. (2.29)) with $G \rightarrow \bar{G}$ and $m \rightarrow \bar{m}$.

Let us then compare a treatment based on the $3 \mathrm{M}$ regularization in terms of the usual variables with the 'extended LB regularization' in terms of LC variables. We treat the constituent quark mass $M$ as a free parameter, choosing the same value in both regularization schemes. Since the expressions for $f_{\pi}$ are the same in the two treatments, we obtain the same value for $\Lambda$ in both schemes by requiring that the experimental value for $f_{\pi}$ is reproduced. 
Then the 4-fermi coupling constant is determined by requiring that the experimental pion mass is reproduced. In the $3 \mathrm{M}$ scheme this is expressed as $1+2 G \Pi_{3 M}\left(m_{\pi}^{2}\right)=0$, while in the LB scheme we have $1+2 \bar{G} \bar{\Pi}_{L B}\left(m_{\pi}^{2}\right)=0$. Since $\bar{\Pi}_{L B}=\Pi_{3 M}$, this gives $G_{3 M}=\bar{G}_{L B}$. Then the gap equation can be used in order to determine the value of the current quark mass. As is clear from the above discussion following eq. (3.54), this gives $\bar{m}_{L B}=m_{3 M}$. Therefore, the current quark masses $(m)$ are different in the two schemes, but also the quark condensates are different: ¿From (3.42) and (3.41), as well as from $\bar{m}_{L B}=m_{3 M}$ we have

$$
\begin{aligned}
\langle\bar{\psi} \psi\rangle_{L B} & =\langle\bar{\psi} \psi\rangle_{3 M}+M F_{L B} \\
m_{L B} & =\frac{m_{3 M}}{1-2 G_{3 M} F_{L B}} .
\end{aligned}
$$

As a consequence the GOR relation holds in both schemes: In the 3M scheme we have eq. (2.35) with $m=m_{3 M}$, and following the same steps in the extended LB scheme we obtain

$$
-m_{L B}\langle\bar{\psi} \psi\rangle_{L B}=f_{\pi}^{2} m_{\pi}^{2}\left(1-\frac{m_{L B}}{M}\right)\left(1+m_{\pi}^{2} C\right)
$$

This 'invariance' of the product of the current quark mass and the quark condensate holds also for $m\langle H|\bar{\psi} \psi| H\rangle$, where $|H\rangle$ denotes a hadronic state, and means that in actual calculations the regularized form $F_{L B}$ and the value of $m$ in the LB scheme are never needed: From the Feynman-Hellman theorem we have $\langle H|\bar{\psi} \psi| H\rangle=\frac{\partial M_{H}}{\partial M} \frac{\partial M}{\partial m}$. The second factor in the latter expression represents the 'vacuum contribution', and its product with $m$ can be written in the form (see Appendix B)

$$
m \frac{\partial M}{\partial m}=\frac{m}{1-2 G \Pi_{\sigma}(0)}=\frac{\bar{m}}{1-2 \bar{G} \bar{\Pi}_{\sigma}(0)},
$$


where $\Pi_{\sigma}(k)$ is the bubble graph in the $\sigma$ meson channel, and $\Pi_{\sigma}(k)=$ $\bar{\Pi}_{\sigma}(k)-F$. ¿From our above discussion it is then clear that the product (3.58), and therefore also the ' $\sigma$-term' $m\langle H|\bar{\psi} \psi| H\rangle$, takes the same value in the ET treatment based on the 3M cut-off and in the LC theory based on the extended LB cut-off.

The above discussion shows that, although the parameters $G$ and $m$ in the LC theory based on the extended LB cut-off scheme are different from those of the ET theory based on the 3M cut-off scheme, the physical predictions are the same. In this sense, the parameters $\bar{G}$ and $\bar{m}$ introduced in the LC theory by eqs. (3.21) and (3.45) can be considered as 'renormalized' parameters, which take the same values as $G$ and $m$ in the ET theory. (The actual value of the 'renormalization constant' $1+2 G F$ is never needed.)

We conclude that the extended LB scheme is physically equivalent to the $3 \mathrm{M}$ scheme.

\subsubsection{Transverse (TR) cut-off scheme}

In this scheme [23], one notes that the divergence of the bubble graph $\bar{\Pi}$ with respect to the longitudinal momentum integration is logarithmic (see (2.22) and (2.23) $)$, and therefore one subtraction is sufficient to make it finite. As a consequence, the following equations are already finite with respect to the longitudinal momentum integration: (i) The pion pole condition written in the form (3.44), (ii) the pionic t-matrix (3.36) written in the form

$$
\bar{\tau}(k)=\frac{-i}{\bar{\Pi}(k)-\bar{\Pi}\left(m_{\pi}^{2}\right)},
$$

(iii) the pion decay constant, and (iv) the quark distribution function. The TR scheme now consists in introducing a transverse momentum cut-off $\Lambda_{T R}$ 
in these equations, while the remaining divergent equations (the gap equation (3.43), and eq. (3.34) determining the quantity $F$ ) are not used explicitly.

Note that in this method the gap equation is used only to formally rewrite the pion pole condition into the form $(\overline{3.44})$, which is finite with respect to the longitudinal momentum integration. It is easy to show that this method works also for the sigma meson channel, i.e; the gap equation can be used to rewrite the equation for the sigma meson mass into a form which is finite with respect to the longitudinal momentum integration. It is a disadvantage of this method, however, that it works strictly only within the present $1 / N$ expansion scheme: The gap equation and the eigenvalue equation for the meson mass must be of the same order in $1 / N$. For the lagrangian (2.1), this is true only for the pion and sigma meson, since the interaction in other mesonic channels is due to the exchange term and suppressed by one power of $1 / N$. It is, nevertheless, possible to apply the TR scheme also to these cases by performing a kind of mass renormalization, that is to use the eigenvalue equation for the meson mass only in a formal sense to rewrite the t-matrix into a renormalized form analogous to (3.59), which is finite with respect to the longitudinal momentum integration. Such a renormalization procedure, in which the gap equation and the eigenvalue equations for the hadron masses are used only in a formal sense, is quite different in spirit from the common usage of the NJL model. 


\section{Numerical results for the quark LC mo- mentum distribution in the pion}

In this section we present numerical results for the quark LC momentum distribution (2.26) regularized by using the two regularization schemes discussed in subsect. 3.5, i.e; the extended LB scheme and the TR scheme. We choose the constituent quark mass $M=300 \mathrm{MeV}$ in both cases. Then by requiring that (2.31) (or (2.38) with the condition (2.36)) reproduces the experimental value $f_{\pi}=93 \mathrm{MeV}$, we obtain for the LB cut-off $\Lambda=1.47 \mathrm{GeV}$ (corresponding to a 3-momentum cut-off of $\left.\Lambda_{3}=0.67 G e V\right)$. I Similarly, for the TR scheme we use (2.38) with $\left|\boldsymbol{q}_{\perp}\right|<\Lambda_{T R}$ to obtain $\Lambda_{T R}=0.48 \mathrm{GeV}$. The value of the pion-quark coupling constant, defined at $k^{2}=m_{\pi}^{2}=(140 \mathrm{MeV})^{2}$, becomes $g=3.15$ (3.16) in the LB (TR) scheme. (These values are close to each other, since in the chiral limit we have $g=M / f_{\pi}=3.23$ in both schemes.)

The resulting distributions multiplied by $x$ are shown by the dashed lines in fig. 1 for the LB scheme, and in fig. 5 for the TR scheme. Due to the condition (2.36) in the LB regularization, the distribution $q_{v}(x)$ is nonzero only in the interval $x_{1}<x<x_{2}$ and tends to zero smoothly at the end points $x_{1,2}$. In the TR regularization, on the contrary, $q_{v}(x)$ extends over the whole interval $0 \leq x \leq 1$ and is nonzero at the end points. In this case, due to the smallness of the pion mass, $q_{v}(x)$ shows only little variation as a function of $x$, which leads to the almost linear behaviour of $x q_{v}(x)$ shown in fig. 5. This is very similar to the result of a previous calculation [8] using the Pauli-Villars regularization based on Feynman integral representation of $q_{v}(x)$ (see the discussion below eq.(2.28)).

\footnotetext{
${ }^{9}$ For the LB scheme, we obtain $G_{3 M}=\bar{G}_{L B}=4.56 \mathrm{GeV}^{-2}$ from the pion pole condition (using $m_{\pi}=140 \mathrm{MeV}$ ), and $m_{3 M}=\bar{m}_{L B}=5.3 \mathrm{MeV}$ from the gap equation.
} 
To connect these results with experiment, we assume as usual [10] that our NJL results are valid at some low-energy scale $Q_{0}^{2}$, and perform the $Q^{2}$ evolution using the DGLAP equations [27] up to the value $Q^{2}=4 \mathrm{GeV}^{2}$, where parametrized fits to the experimental results are available [24]. We used the computer code of ref. [28] to solve the DGLAP equations up to the next-to-leading order, using the parameters $\Lambda_{Q C D}=0.25 \mathrm{GeV}$ and $N_{f}=3$. The value of $Q_{0}^{2}$ is considered here as a parameter to be adjusted such as to reasonably reproduce the experimental distribution. In both regularization schemes the value obtained in this way is $Q_{0}^{2}=0.18 \mathrm{GeV}^{2}$, i.e; $Q_{0}$ is of the order of the constituent quark mass.

The results of the $Q^{2}$ evolution are shown by the solid lines in figs. Tt and 5, 四 and are compared to the empirical distribution given in ref. [24] (dotted lines). The fact that in the LB scheme the theoretical distribution is too low for high $x$ is connected to the LB condition (2.36) which gives a vanishing input distribution for high $x$ (see the dashed line in fig. 4). The use of smaller values for $Q_{0}^{2}$ would lead to better agreement around the peak position, but to a larger discrepancy for higher $x$. A similar situation is encountered also in the case of the nucleon structure function [29]. On the other hand, the result based on the TR scheme reproduces the empirical distribution almost exactly. Therefore, from the phenomenological point of view, a regularization scheme which does not restrict the longitudinal momenta seems to be favorable.

\footnotetext{
${ }^{10}$ Since the computer code used for the $Q^{2}$ evolution $[28$ requires an input distribution $x q_{v}(x)$ which vanishes at $x=1$, we artificially modified it for $x$ very close to 1 such that it goes like $(1-x)^{n}$ with some power $n$. ( $n=10$ was used in the actual calculation.)
} 


\section{Summary and conclusions}

In this work we investigated the NJL model in the LC quantization and its relation to the usual ET formalism, putting special emphasis on the application to the pion structure function. We first reviewed the NJL model and the calculation of the pion structure function in the ET formalism. We showed that the Lepage-Brodsky (LB) cut-off scheme for the quark LC momentum distribution can be applied consistently in connection with the covariant 3momentum cut-off (3M) scheme for the gap equation and the $q \bar{q}$ polarization propagator.

The introduction of LC variables in the ET formalism is simply a transformation of variables. As we discussed in the introduction, however, the interpretation of the structure function as LC momentum distributions of the constituents in the ground state target is most natural in the LC quantization procedure. Due to this fact, and other physically appealing features of the LC formalism, we formulated the LC quantization of the NJL model. The essential part was the solution of the fermionic constraint equation using the $1 / N$ expansion up to the next-to-leading order. We showed that, up to this order, the interaction lagrangian on the LC for the dynamical component of the fermion field has the same form as obtained by using the free field equations to solve the fermionic constraint, but the original 4-fermi coupling constant is replaced by an effective (renormalized) one. The Feynman rules for the LC lagrangian are very similar to those for the ET lagrangian: The propagators for internal lines differ just by a contact term, and the 4-fermi vertices differ by a multiplying factor. For physical quantities these differences formally cancel with each other, leading to the same results as in the ET 
theory. We have demonstrated this equivalence for the eigenvalue equation determining the pion mass, the pion decay constant, and the pion structure function in the Bjorken limit. In the LC quantization, these quantities can be evaluated either by using the language of Feynman diagrams as described above, or by using the Fock space expansions based on the hamiltonian formalism. Both methods lead to the same results.

In this work, we avoided the difficulty of regularizing tadpole-like diagrams in the LC theory by relating them to 2-point Green functions at zero momentum. We then formulated two possible regularization schemes: The first one was an extension of the "invariant mass cut-off scheme" [21]. We have shown that this scheme leads to the same physical predictions as the covariant 3-momentum cut-off (dispersion cut-off) scheme in the ET formulation, provided that in both treatments the parameters are fixed such as to have the same values for $M, f_{\pi}$, and $m_{\pi}$. The application of this scheme to problems others than studied in this paper, like the nucleon structure function, is straight forward [29]. The second one ("transverse cut-off scheme") employs a cut-off only for the transverse momentum components. In this scheme, the gap equation is used only to formally rewrite the eigenvalue equation for the pion and sigma mass into a form which is finite with respect to the longitudinal momentum integrations. We noted that this method works only within the present $1 / N$ expansion scheme, and to apply it to other meson channels or to the nucleon, one has in addition to perform a kind of mass renormalization. Such a procedure, however, departs significantly from the common usage of the NJL model.

As an application, we evaluated the quark LC momentum distribution in the pion numerically and performed the $Q^{2}$ evolution to compare the re- 
sults with parametrizations based on experimental data. Since in the LB regularization scheme the input distribution is zero in an interval near the end points, the slope of the evolved distribution on the large $x$ side is somewhat too large compared with the empirical distribution. Nevertheless, a fair agreement can be achieved by assigning a low energy scale $Q_{0}^{2}=0.18 \mathrm{GeV}^{2}$ to the NJL model, which is similar to the values used in previous investigations of the pion structure function. On the other hand, in the TR regularization scheme one can reproduce the empirical distribution almost exactly using the same value of $Q_{0}^{2}$. This indicates that for phenomenological applications a regularization scheme which does not restrict the longitudinal momentum components seems to be preferable.

\section{ACKNOWLEDGEMENTS}

The authors would like to thank M. Miyama and S. Kumano for the computer program used for the $Q^{2}$ evolution (ref. [28]). One of the authors (W.B.) is grateful to A. W. Thomas and A. W. Schreiber for discussions on the pion structure function, and to M. Burkardt and K. Itakura for discussions on light cone theories. 


\section{Appendices}

\section{A LC variables and Dirac matrices}

In this Appendix we summarize our conventions for LC variables and Dirac matrices, and give some useful identities which have been used in the main

text. We use the LC variables $a^{ \pm}=\frac{1}{\sqrt{2}}\left(x^{0} \pm x^{3}\right), a_{ \pm}=\frac{1}{\sqrt{2}}\left(x_{0} \pm x_{3}\right)$, and $x_{\perp}^{i}=-x_{\perp i}(i=1,2)$. Then we have for the Lorentz scalar product $p \cdot x=$ $p_{+} x^{+}+p_{-} x^{-}-\boldsymbol{p}_{\perp} \cdot \boldsymbol{x}_{\perp}$. In the LC quantization procedure, $x^{+}$is the 'time' variable and $x^{-}, \boldsymbol{x}_{\perp}$ are the 'space' variables. Similarly, $p_{+}$is the 'energy' variable and $p_{-}, \boldsymbol{p}_{\perp}$ are the 'momentum' variables. The dispersion relation for a free particle with mass $M$ is $p_{+} \equiv e_{p}=\left(\boldsymbol{p}_{\perp}^{2}+M^{2}\right) / 2 p_{-}$, see eq. (2.19). For the derivatives we use the conventions $\partial_{a}=\partial / \partial x^{a}(a=+,-, 1,2)$.

Analogous definitions hold for the LC Dirac matrices $\gamma^{ \pm}, \gamma_{\perp}$ in terms of the $\gamma^{\mu}$. It follows that $\not \partial=\gamma^{+} \partial_{+}+\gamma^{-} \partial_{-}-\gamma_{\perp} \cdot \partial_{\perp}$. Note that $\gamma^{+} \gamma^{+}=$ $\gamma^{-} \gamma^{-}=0$. The projection operators $\Lambda_{ \pm}$have been introduced in eq. (3.1). Equivalent useful forms are $\Lambda_{ \pm}=\frac{1}{\sqrt{2}} \gamma^{0} \gamma^{ \pm}=\frac{1}{\sqrt{2}} \gamma^{\mp} \gamma^{0}=\frac{1}{2}\left(1 \pm \gamma^{0} \gamma^{3}\right)$. We have the following useful identities:

$$
\begin{array}{r}
\gamma^{-} \Lambda_{+}=\Lambda_{+} \gamma^{+}=\gamma^{+} \Lambda_{-}=\Lambda_{-} \gamma^{-}=0 \\
\Lambda_{ \pm} \gamma^{0}=\gamma^{0} \Lambda_{\mp}
\end{array}
$$

For the quark bilinears it follows that

$$
\begin{aligned}
\bar{\psi} \psi & =\psi_{+}^{\dagger} \gamma^{0} \psi_{-}+\psi_{-}^{\dagger} \gamma^{0} \psi_{+} \\
\bar{\psi} \gamma^{ \pm} \psi & =\sqrt{2} \psi_{ \pm}^{\dagger} \psi_{ \pm} \\
\bar{\psi} \gamma_{\perp} \cdot \boldsymbol{\partial}_{\perp} \psi & =\psi_{+}^{\dagger} \boldsymbol{\alpha}_{\perp} \cdot \boldsymbol{\partial}_{\perp} \psi_{-}+\psi_{-}^{\dagger} \boldsymbol{\alpha}_{\perp} \cdot \boldsymbol{\partial}_{\perp} \psi_{+}
\end{aligned}
$$


We also give some identities which were used in deriving eqs. (3.29) and (3.50):

$$
\begin{aligned}
\Lambda_{ \pm}(\not p+M) \Lambda_{ \pm} & =\Lambda_{ \pm}\left(-\gamma_{\perp} \cdot \boldsymbol{p}_{\perp}+M\right) \\
\Lambda_{ \pm}(\not p+M) \gamma^{0} \Lambda_{ \pm} & =\sqrt{2} \Lambda_{ \pm} p_{\mp} \\
\Lambda_{ \pm}(\not p+M) \Lambda_{\mp} & =\gamma^{\mp} p_{\mp} \\
\Lambda_{ \pm}(\not p+M) \gamma^{0} \Lambda_{\mp} & =\frac{1}{\sqrt{2}} \gamma^{\mp}\left(\boldsymbol{\gamma}_{\perp} \cdot \boldsymbol{p}_{\perp}+M\right) .
\end{aligned}
$$

The Dirac spinors are normalized according to $u^{\dagger}\left(\boldsymbol{p}, s^{\prime}\right) u(\boldsymbol{p}, s)=2 E_{p} \delta_{s^{\prime} s}$ and $v^{\dagger}\left(\boldsymbol{p}, s^{\prime}\right) v(\boldsymbol{p}, s)=2 E_{p} \delta_{s^{\prime} s}$. The spinors $u_{+}=\Lambda_{+} u$ and $v_{+}=\Lambda_{+} v$ then satisfy the orthogonality and completeness relations

$$
\begin{aligned}
u_{+}^{\dagger}\left(\boldsymbol{p}, s^{\prime}\right) u_{+}(\boldsymbol{p}, s) & =v_{+}^{\dagger}\left(\boldsymbol{p}, s^{\prime}\right) v_{+}(\boldsymbol{p}, s)=\sqrt{2} p_{-} \delta_{s s^{\prime}} \\
\sum_{s} u_{+}(\boldsymbol{p}, s) u_{+}^{\dagger}(\boldsymbol{p}, s) & =\sum_{s} v_{+}(\boldsymbol{p}, s) v_{+}^{\dagger}(\boldsymbol{p}, s)=\sqrt{2} \Lambda_{+} p_{-} .
\end{aligned}
$$

\section{B Relations between the condensate and 2- point functions}

We first derive relation (2.32) in the ET formalism from the chiral Ward identity for the pion decay vertex. If we define $J_{A}^{i \mu}=\bar{\psi} \gamma^{\mu} \gamma_{5} \tau_{i} \psi$ and $\pi^{i}=$ $\bar{\psi} i \gamma_{5} \tau_{i} \psi$, the chiral Ward identity follows as usual from the PCAC relation $\partial_{\mu} J_{A}^{i \mu}=2 m \pi^{i}$ :

$$
\begin{aligned}
& \partial_{\mu}\left\langle 0\left|T\left(J_{A}^{i \mu}(x) \pi^{j}\left(x^{\prime}\right)\right)\right| 0\right\rangle \\
& \quad=2 m\left\langle 0\left|T\left(\pi^{i}(x) \pi^{j}\left(x^{\prime}\right)\right)\right| 0\right\rangle-2 i \delta_{i j} \delta^{(4)}\left(x^{\prime}-x\right)\langle 0|\bar{\psi} \psi| 0\rangle .
\end{aligned}
$$

To lowest order in $1 / N$, the correlation function $\left\langle 0\left|T\left(\pi^{i}(x) \pi^{j}\left(x^{\prime}\right)\right)\right| 0\right\rangle$ is given by the series of bubble graphs. Considering the Fourier transform of $(\mathbb{B} .1)$ 
for $k=0$, we therefore obtain in the leading order of $1 / N$

$$
0=2 i m \frac{\Pi(0)}{1+2 G \Pi(0)}-2 i\langle 0|\bar{\psi} \psi| 0\rangle .
$$

Since the constituent quark mass is defined by $M=m-2 G\langle 0|\bar{\psi} \psi| 0\rangle$, this relation can be written in the form $\langle 0|\bar{\psi} \psi| 0\rangle=M \Pi(0)$, which is eq. (2.32).

Next we derive eq. (3.58) from the general relation

$$
\frac{\partial\langle\bar{\psi} \psi\rangle}{\partial M}=-\Pi_{\sigma}(0)
$$

where $\Pi_{\sigma}(k)$ is the bubble graph in the $\sigma$ meson channel. Eq. (B.3) can be derived most easily by using the functional integral. It is easy to check that this relation holds in the $3 \mathrm{M}$ regularization scheme to leading order in $1 / N$, i.e; $\frac{\partial\langle\bar{\psi} \psi\rangle_{3 M}}{\partial M}=-\Pi_{\sigma, 3 M}(0)$. Then, in our extended LB scheme, differentiating (3.55) with respect to $M$ and requiring that the result is equal to $-\Pi_{\sigma, L B}(0)=-\left(\bar{\Pi}_{\sigma, L B}(0)-F_{L B}\right)$ leads to the requirement that $F_{L B}$ is independent of $M$. By differentiating the gap equation (2.3) with respect to $M$ we obtain

$$
m \frac{\partial M}{\partial m}=\frac{m}{1+2 G \frac{\partial}{\partial M}\langle\bar{\psi} \psi\rangle}=\frac{m}{1-2 G \Pi_{\sigma}(0)}=\frac{\bar{m}}{1-2 \bar{G} \bar{\Pi}_{\sigma}(0)},
$$

where we used $\frac{G}{\bar{G}}=\frac{m}{\bar{m}}=\frac{1}{1-2 \bar{G} F}$. This proves eq. (3.58).

\section{Bound state equation}

In this Appendix we derive the bound state equation in the TDA for the LC wave function in the pionic $q \bar{q}$ channel, using (i) the TDA equation in the hamiltonian formalism, and (ii) the BS equation. 


\section{C.1 Hamiltonian formalism}

The LC hamiltonian derived from the lagrangian eq. (3.20) is given in terms of $\psi_{+}$by

$$
\begin{aligned}
H & =\int d x^{-} d^{2} x_{\perp}\left(\psi_{+}^{\dagger}\left(-\boldsymbol{\partial}_{\perp}^{2}+M^{2}\right) \frac{1}{\sqrt{2} i \partial_{-}} \psi_{+}\right. \\
& +\bar{G}\left[: \psi_{+}^{\dagger} \gamma_{5} \gamma^{0} \boldsymbol{\tau}\left\{\frac{-1}{\sqrt{2} i \partial_{-}}\left(i \boldsymbol{\alpha}_{\perp} \cdot \boldsymbol{\partial}_{\perp}+M\right)+\left(i \boldsymbol{\alpha}_{\perp} \cdot \overleftarrow{\boldsymbol{\partial}}_{\perp}-M\right) \frac{1}{\sqrt{2} i \overleftarrow{\partial}_{-}}\right\} \psi_{+}:\right]_{(\text {C. } 1)}^{2} \\
& +\sigma \text { channel, }
\end{aligned}
$$

where we show only the interaction in the pionic channel explicitly. We now insert (3.22) into the above hamiltonian and apply it to the pion state (3.52), keeping only those terms which are again of the form (3.52). (This corresponds to the TDA, and means that in the interaction hamiltonian only terms of the form $b^{\dagger} d^{\dagger} b d$ are kept.) The result is expressed as an eigenvalue equation for the 'energy' $\epsilon_{k}=\left(\boldsymbol{k}_{\perp}^{2}+m_{\pi}^{2}\right) / 2 k_{-}$, i.e; $H|k\rangle=\epsilon_{k}|k\rangle$. The procedure is tedious but straight forward, and therefore we will give only the final result. One ends up with a TDA equation, where the kernel has both direct and exchange terms. The kernel for the direct term leads to a color factor 3 , and in order to be consistent with the leading order in the $1 / N$ expansion used throughout this paper we keep only the direct term. The result is

$$
\begin{aligned}
& \left(\epsilon_{k}-\frac{\boldsymbol{q}_{\perp}^{2}+M^{2}}{2 k_{-} x}-\frac{(\boldsymbol{k}-\boldsymbol{q})_{\perp}^{2}+M^{2}}{2 k_{-}(1-x)}\right) \Phi_{k, a_{1} a_{2}}^{(S) i}(\boldsymbol{q}) \\
& =-\frac{\bar{G}}{6 k_{-}^{3}} \int_{0}^{1} \frac{d x^{\prime}}{\sqrt{x(1-x) x^{\prime}\left(1-x^{\prime}\right)}} \int \frac{d^{2} q_{\perp}^{\prime}}{(2 \pi)^{3}}\left(u_{+}^{\dagger}\left(\boldsymbol{q}, s_{1}\right) \chi^{\dagger}\left(t_{1}\right) \hat{X}_{k}^{i}(\boldsymbol{q}) v_{+}\left(\boldsymbol{k}-\boldsymbol{q}, s_{2}\right)\right. \\
& \left.\times \quad \chi\left(t_{2}\right) \delta_{c_{1} c_{2}}\right)\left(v_{+}^{\dagger}\left(\boldsymbol{k}-\boldsymbol{q}^{\prime}, s_{2}^{\prime}\right) \chi^{\dagger}\left(t_{2}^{\prime}\right) \hat{X}_{k}^{j}\left(\boldsymbol{q}^{\prime}\right) u_{+}\left(\boldsymbol{q}^{\prime}, s_{1}^{\prime}\right) \chi\left(t_{1}^{\prime}\right) \delta_{c_{1}^{\prime} c_{2}^{\prime}}\right) \Phi_{k, a_{1}^{\prime} a_{2}^{\prime}}^{(S)}\left(\boldsymbol{q}^{\prime}\right)
\end{aligned}
$$


with (see eq. (3.25))

$$
\hat{X}_{k}^{i}(\boldsymbol{q})=k_{-} X_{k}^{i}(\boldsymbol{q})=\gamma_{5} \tau_{i}\left(\frac{\boldsymbol{\gamma}_{\perp} \cdot \boldsymbol{q}_{\perp}+M}{\sqrt{2} x}+\frac{\gamma_{\perp} \cdot(\boldsymbol{q}-\boldsymbol{k})_{\perp}+M}{\sqrt{2}(1-x)}\right) .
$$

Here the variables $x$ and $x^{\prime}$ have been introduced according to $q_{-}=k_{-} x$ and $q_{-}^{\prime}=k_{-} x^{\prime}$. The notation is as in eq. (3.50), i.e; $a_{i}=\left(s_{i}, t_{i}, c_{i}\right)$. It is clear that for $\boldsymbol{k}_{\perp}=0$ the solution of this equation is of the form (3.50). By inserting this form back into (C.2) we get the eigenvalue equation for $\epsilon_{k}$ which coincides with the equation $1+2 \bar{G} \bar{\Pi}(k)=0$ with $\bar{\Pi}(k)$ given by eq. (2.23).

\section{C.2 BS equation}

The BS wave function for the dynamical components of the fermion field has been defined in eq.(3.47) and satisfies the following BS equation:

$\tilde{\Phi}_{k, \alpha \beta}^{i}(q)=\frac{2 i \bar{G}}{k_{-}^{2}} \int \frac{d^{4} q^{\prime}}{(2 \pi)^{4}}\left(\bar{S}_{++}(q) \hat{X}_{k}^{i}(\boldsymbol{q}) \bar{S}_{++}(q-k)\right)_{\alpha \beta} \frac{1}{3} \operatorname{Tr}\left(\hat{X}_{k}^{j}\left(\boldsymbol{q}^{\prime}\right) \tilde{\Phi}_{k}^{j}\left(q^{\prime}\right)\right)$,

where the propagator $\bar{S}_{++}$is given in eq. (3.23), and the interaction kernel (3.25) has been expressed in terms of the quantity $\hat{X}$ of (C.3), where $q_{-}=k_{-} x$ and $q_{-}^{\prime}=k_{-} x^{\prime}$. We integrate eq. (C.4) over $q_{+}$to derive the equation for the wave function $\Phi_{k}^{(S)}(\boldsymbol{q})$ defined in eq. 3.49 ):

$$
\begin{aligned}
& \left(k_{+}-\frac{\boldsymbol{q}_{\perp}^{2}+M^{2}}{2 k_{-} x}-\frac{(\boldsymbol{k}-\boldsymbol{q})_{\perp}^{2}+M^{2}}{2 k_{-}(1-x)}\right) \Phi_{k, \alpha \beta}^{(S) i}(\boldsymbol{q}) \\
& =-\frac{\bar{G}}{k_{-}} \int_{0}^{1} d x^{\prime} \int \frac{d^{2} q_{\perp}^{\prime}}{(2 \pi)^{3}} \hat{X}_{k, \alpha \beta}^{i}(\boldsymbol{q}) \frac{1}{3} \operatorname{Tr}\left(\hat{X}_{k}^{j}\left(\boldsymbol{q}^{\prime}\right) \Phi_{k}^{(S) j}\left(\boldsymbol{q}^{\prime}\right)\right) .
\end{aligned}
$$


The corresponding wave function in the spin representation (see the discussion below eq. (3.49))

$$
\Phi_{k, a_{1} a_{2}}^{(S) i}(\boldsymbol{q})=\frac{1}{k_{-} \sqrt{2 x(1-x)}} u_{+}^{\dagger}\left(\boldsymbol{q}, s_{1}\right) \chi^{\dagger}\left(t_{1}\right) \Phi_{k}^{(S) i}(\boldsymbol{q}) v_{+}\left(\boldsymbol{k}-\boldsymbol{q}, s_{2}\right) \chi\left(t_{2}\right) \delta_{c_{1} c_{2}}
$$

then satisfies the equation (C.2).

\section{Bound state matrix elements using the LC BS wave functions}

If we use the BS wave function $\bar{\Phi}_{k}$ in the diagram for pion decay, referring to the + component of the axial vector current, the resulting expression differs from eq. (2.10) only by the replacement $S \rightarrow \bar{S}$ for the two quark lines. (Note that the + component of the axial vector current can be expressed as $\bar{\Psi} \gamma^{+} \gamma_{5} \tau \Psi$.) Due to relation (2.20) it is then easy to see that this replacement

leads to no changes. If we use the wave function $\tilde{\Phi}_{k}$, the trace factor involves two extra factors $\Lambda_{+}$, but due to the relation $\gamma^{0} \gamma^{+}=\sqrt{2} \Lambda_{+}$these factors can be left out, leading again to the same result.

If we use the BS wave function $\bar{\Phi}_{k}$ together with the propagators $\bar{S}$ in the diagram fig. 2 for the pion structure function, the resulting expression differs from eq. 2.13) only by the replacement $S \rightarrow \bar{S}$ for all three quark lines. From (2.20) and the familiar relation $S(q) \gamma^{0} \gamma^{+} S(q)=-\partial S(q) / \partial q_{+}$ it follows that also $\bar{S}(q) \gamma^{0} \gamma^{+} \bar{S}(q)=-\partial \bar{S}(q) / \partial q_{+}$, and performing a partial integration in $q_{+}$we end up with $q_{v}(x)=-g^{2} \partial \bar{\Pi}(k, x) / \partial k^{2}$. Due to relation (2.21) this is the same as (2.14). If we calculate the diagram fig. 2 by using 
the wave function $\tilde{\Phi}_{k}$ and the propagators (3.23), the trace factor is

$$
\operatorname{Tr}\left[\Lambda_{+} \bar{S}(q) \gamma^{0} \gamma_{5} \bar{S}(q-k) \Lambda_{+} \bar{S}_{++}^{-1}(q-k) \Lambda_{+} \bar{S}(q-k) \gamma^{0} \gamma_{5} \bar{S}(q) \Lambda_{+} \gamma^{0} \gamma_{5}\right]
$$

Using the identity (see eq. (3.26) and (3.29) )

$\bar{S}(q-k)\left(\Lambda_{+} \bar{S}_{++}^{-1}(q-k) \Lambda_{+} \bar{S}(q-k)\right)=\bar{S}(q-k) \Lambda_{+}(1+Z(\boldsymbol{q}-\boldsymbol{k}))=\bar{S}(q-k)$

and $\gamma^{0} \gamma^{+}=\sqrt{2} \Lambda_{+}$, the result is the same as obtained by calculating the diagram fig. 2 by using the propagators $\bar{S}$. As was shown above, this gives the result (2.14) of the ET theory.

\footnotetext{
${ }^{11}$ The quantity $\bar{S}_{++}^{-1}$ in eqs. (D.1), (D.2) is understood as the inverse of eq. (3.23) except for the projection operator $\Lambda_{+}$.
} 


\section{References}

[1] Y. Nambu and G. Jona-Lasinio, Phys. Rev. 122 (1960) 345; 124 (1961) 246.

[2] R. Brockmann, W. Weise and E. Werner, Phys. Lett. B 122 (1983) 201;

D. Ebert and H. Reinhardt, Nucl. Phys. B 271 (1986) 188;

U. Vogl and W. Weise, Prog. Part. Nucl. Phys. 27 (1991) 195;

M. Kato, W. Bentz, K. Yazaki and K. Tanaka, Nucl. Phys. A 551 (1993) 541.

[3] N. Ishii, W. Bentz and K. Yazaki, Phys. Lett. B 301 (1993) 165; B 318 (1993) 26;

N. Ishii, W. Bentz and K. Yazaki, Nucl. Phys. A 578 (1995) 617.

[4] U. Zückert, R. Alkofer, H. Weigel and H. Reinhardt, Phys. Rev. 55 (1997) 2030;

S. Huang and J. Tjon, Phys. Rev. C 49 (1994) 1702;

C. Hanhart and S. Krewald, Phys. Lett. B 344 (1995) 55.

[5] N. Ishii, Phys. Lett. B 431 (1998) 1.

[6] A. Bender, C.D. Roberts and L.v. Smekal, Phys. Lett. B 380 (1996) 7; G. Hellstern, R. Alkofer, M. Oettel and H. Reinhardt, Nucl. Phys. A 627 (1997) 679.

[7] M. Wakamatsu and H. Yoshiki, Nucl. Phys. A 542 (1991) 561;

H. Asami, N. Ishii, W. Bentz and K. Yazaki, Phys. Rev. C 52 (1996) 3388. 
[8] T. Shigetani, K. Suzuki and H. Toki, Phys. Lett. B 308 (1993) 383;

R.M. Davidson and E. Ruiz Arriola, Phys. Lett. B 348 (1995) 163;

C.M. Shakin and Wei-Dong Sun, Phys. Rev. C 53 (1996) 3152.

[9] H. Weigel, L. Gamberg and H. Reinhardt, Mod. Phys. Lett. A 11 (1996) 3021 ;

M. Wakamatsu and T. Kubota, Chiral symmetry and the nucleon spin structure functions, hep-ph/9809443.

[10] R.L. Jaffe, 1985 Los Alamos School on Relativistic Dynamics and Quark Nuclear Physics, eds. M.B. Johnson and A. Pickleseimer, Wiley, New York, 1985.

[11] M. Burkardt, Adv. Nucl. Phys. 23 (1996) 1.

[12] S.-J. Chang, R. G. Root and T.-M. Yan, Phys. Rev. D 7 (1973) 1133;

S.-J. Chang and T.-M. Yan, Phys. Rev. D 7 (1973) 1147;

T.-M. Yan, Phys. Rev. D 7 (1973) 1760; D 7 (1973) 1780.

[13] F. Lenz, M. Thies, S. Levit and K. Yazaki, Ann. Phys. (N.Y.) 208 (1991) 1.

[14] P.A.M. Dirac, Rev. Mod. Phys. 21 (1949) 392.

[15] H. Leutwyler, J.R. Klauder and L. Streit, Nuovo Cim. A 66 (1970) 536.

[16] H.-C. Pauli and S.J. Brodsky, Phys. Rev. D 32 (1985) 1993; D 32 (1985) 2001;

R. J. Perry, A. Harindranath and K.G. Wilson, Phys. Rev. Lett. 65 (1990) 2959. 
[17] K. Yamawaki, Zero mode problem on the light front, hep-th/9802037;

D.G. Robertson, The Vacuum in light-cone field theory, hep-th/9603205;

K. Harada, A. Okazaki and M. Taniguchi, Phys. Rev. D 55 (1997) 4910.

[18] K. Itakura, Toward a description of broken phases in light-front field theories. Ph.D. thesis, Univ. of Tokyo (1996), unpublished;

K. Itakura, Prog. Theor. Phys. 98 (1997) 527.

[19] C. Dietmaier, T. Heinzl, M. Schaden and E. Werner Z. Phys. A 334 (1989) 215.

[20] T. Hama, The Nambu-Jona-Lasinio model on the light cone and its application to structure functions. Master thesis, Univ. of Tokyo (1998), unpublished.

[21] G.P. Lepage and S.J. Brodsky, Phys. Rev. D 22 (1980) 2157.

[22] T. Hatsuda and T. Kunihiro, Phys. Rep. 247 (1994) 221.

[23] F. Lenz, K. Ohta, M. Thies and K. Yazaki, to be published.

[24] P.J. Sutton, A.D. Martin, R.G. Roberts and W.J. Stirling, Phys. Rev. D 45 (1992) 2349.

[25] V. Bernard, R. Brockmann, M. Schaden, W. Weise and E. Werner, Nucl. Phys. A 412 (1984) 349.

[26] B. Chibisov and A. Zhitnitsky, Phys. Lett. B 362 (1995) 105;

M. Burkardt, Phys. Rev. D 53 (1996) 933;

T. Heinzl, Phys. Lett. B 388 (1996) 129. 
[27] V.N. Gribov and L.N. Lipatov, Sov. J. Nucl. Phys. 15 (1972) 438 and 675 ;

G. Altarelli and G. Parisi, Nucl. Phys. B 126 (1977) 298;

Yu.L. Dokshitzer, Sov. Phys. JETP 46 (1977) 641.

[28] M. Miyama and S. Kumano, Comp. Phys. Commun. 94 (1996) 185.

[29] H. Mineo, W. Bentz and K. Yazaki, Nucleon structure function in the NJL model, in preparation;

W. Bentz, H. Mineo, N. Ishii, H. Asami, and K. Yazaki, Relativistic Faddeev description of baryons and nucleon structure function in the NJL model, the 1998 YITP Workshop on QCD and Hadron Physics (Oct. 1998), to be publ. by World Scientific, Singapore (1999). 


\section{Figure captions}

1. External momenta for the $q \bar{q}$ t-matrix.

2. Diagram for the quark LC momentum distribution in the pion. The double line represents the pion, the shaded area the pionic vertex function, and cross represents the external operator $\gamma^{+}$.

3. Vertex correction to the 'bare' vertex (the cross in fig. 2).

4. LC momentum distributions of the valence quark in the pion, using the LB regularization scheme. The dashed line shows the NJL model result (eq. (2.26) with the condition (2.36) ) with the parameters $M=0.3 \mathrm{GeV}$ and $\Lambda=1.47 \mathrm{GeV}$. The solid line shows the result obtained by the QCD evolution up to next-to-leading order from $Q_{0}^{2}=0.18 \mathrm{GeV}^{2}$ to $Q^{2}=4 G e V^{2}$, using $\Lambda_{Q C D}=0.25 G e V$ and $N_{f}=3$. The dotted line shows the parametrization at $Q^{2}=4 G e V^{2}$ obtained in ref. [24] by analyzing the experimental data.

5. LC momentum distributions of the valence quark in the pion, using the TR regularization scheme. The dashed line is calculated by using the cut-off $\Lambda_{T R}=0.48 \mathrm{GeV}$ for the transverse momentum integration in eq. (2.26). For further explanation, see the caption to fig. 1 . 

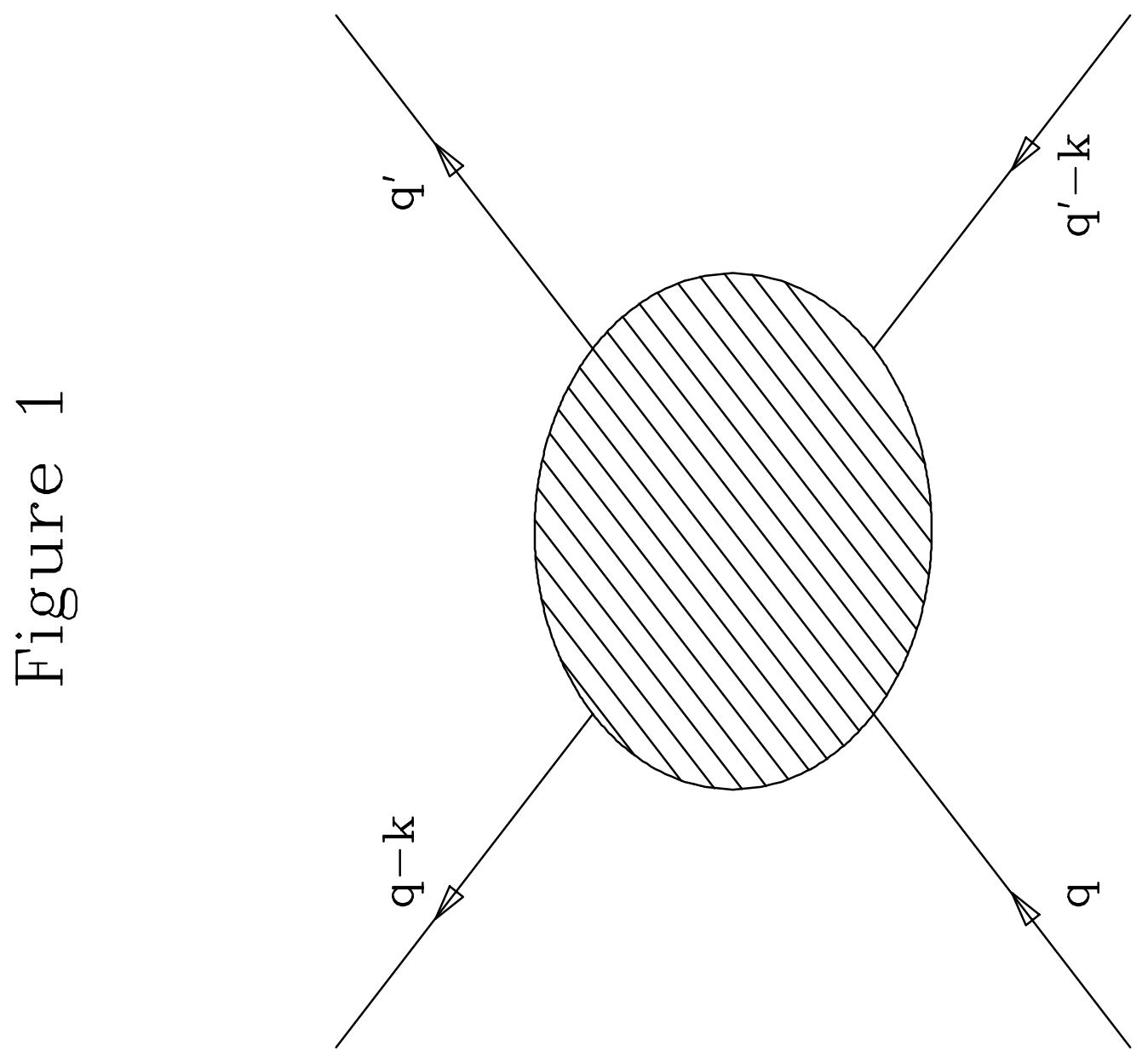

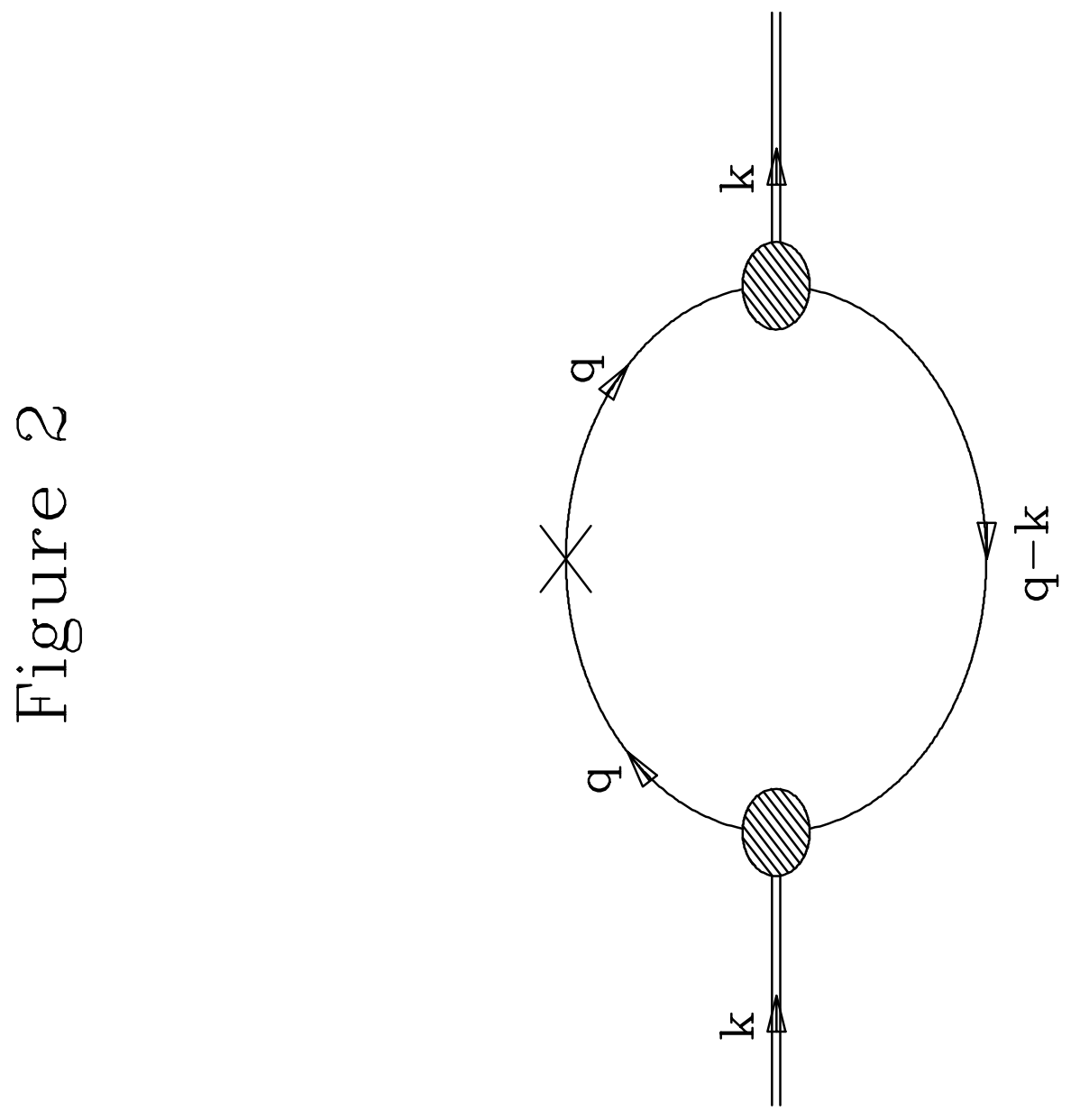

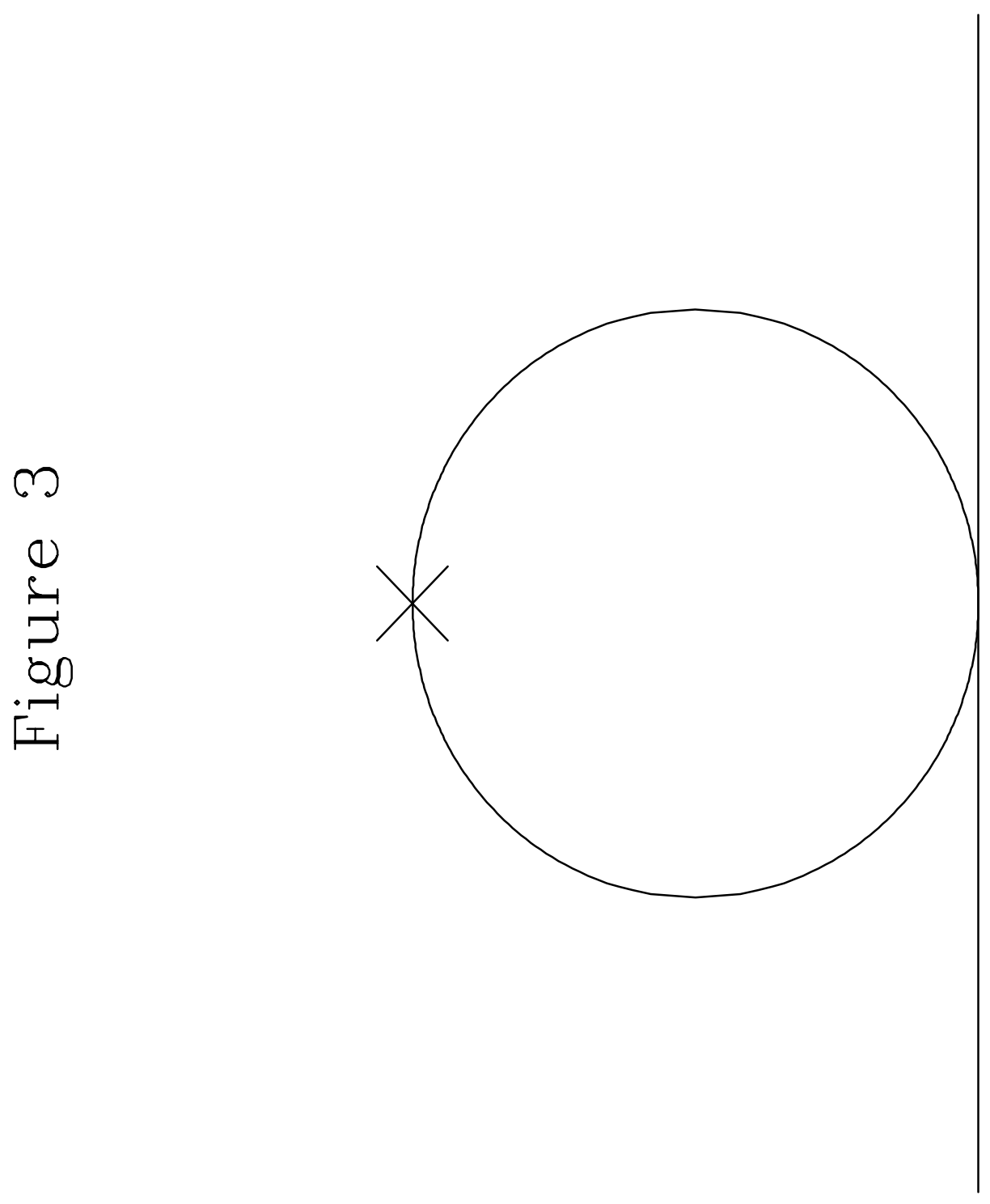


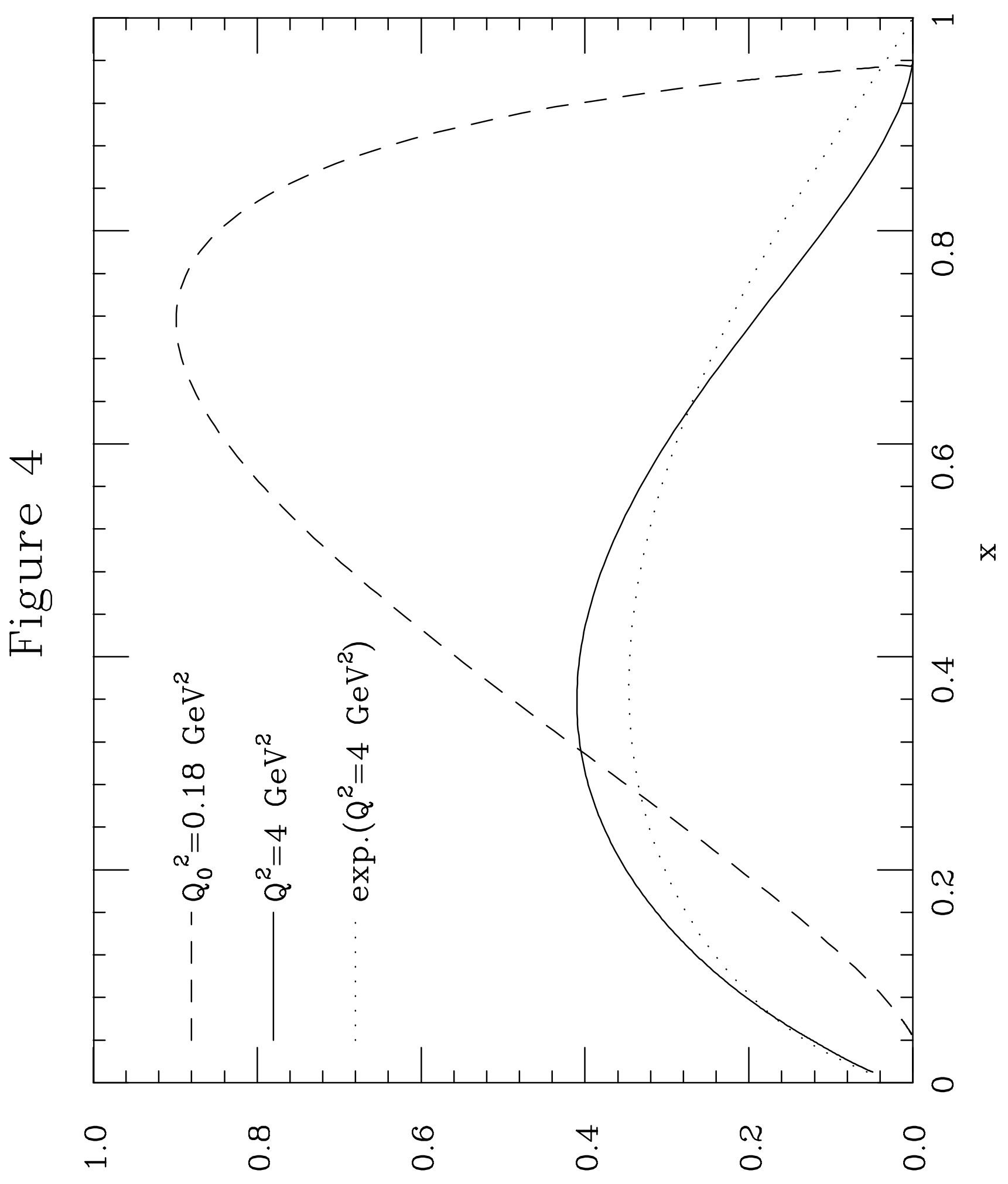

${ }^{\wedge} \mathrm{bx}$ 


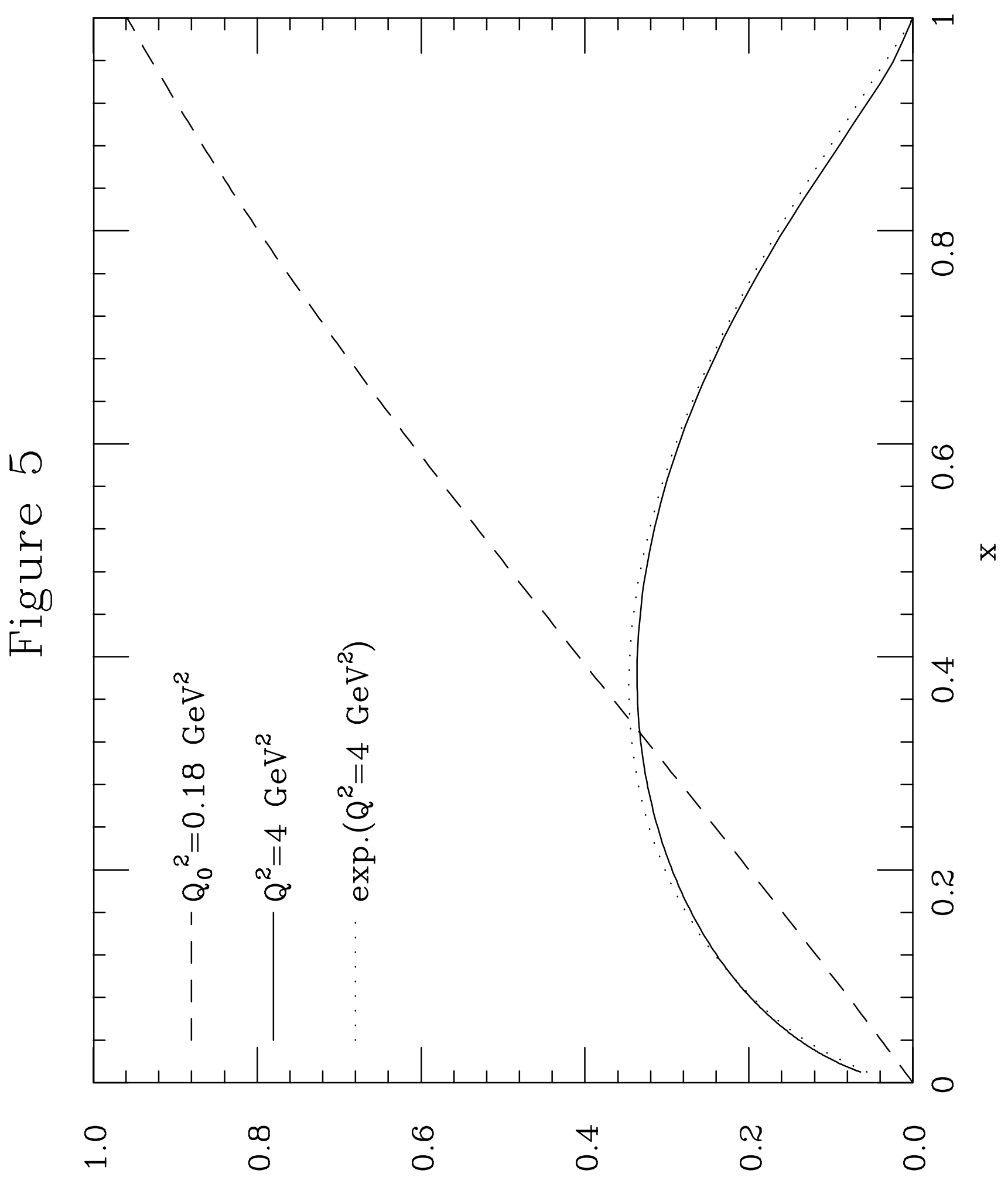

${ }^{\wedge} \mathrm{bx}$ 